\title{
REFLECTION-REFRACTION COEFFICIENTS AND ENERGY RATIOS IN COUPLE STRESS MICROPOLAR THERMOVISCOUS ELASTIC SOLID
}

\author{
Ravinder KUMAR SAHRAWAT and POONAM* \\ Department of Mathematics, Deenbandhu Chhotu Ram University of Science and Technology \\ Murthal-131039, INDIA \\ E-mail: poonamsangwan2793@gmail.com
}

\begin{abstract}
The reflection and refraction phenomenon of propagation of waves in couple stress micropolar thermoviscous elastic solid media with independent viscoelastic and micropolar properties have been studied. The structure of the model has been taken such that the plane interface is divides the given media into two half spaces in perfect contact. Here, we find that there are five waves, one of them is propagating independently while others are set of two coupled waves travelling with different speeds. Energy ratios, reflection and refraction coefficients relative to numerous reflected and refracted waves have been investigated when set of two coupled longitudinal waves and set of two coupled transverse waves strike at the interface through the solid medium. The inequality of energy ratios, refraction coefficients and reflection coefficients are evaluated numerically and presented graphically under three theories of thermoelasticity, namely, Green-Lindsay theory (GL), Lord- Shulman theory (LS), Coupled theory (CT) versus angular frequency and angle of incidence.
\end{abstract}

Key words: micropolar, thermoviscous, phase speed, energy ratio, reflection coefficient, refraction coefficient, couple stress.

\section{Introduction}

The micropolar theory developed by Eringen [1]. It is helpful in many structured materials like lattice micropolar structure. This theory explains the variation of micropolar elastic materials from classic elastic materials which are independent of translation. Eringen [2] and Nowacki [3] elaborated the micropolar thermoelasticity theory. The generalized micropolar theory was introduced by Dost and Taborrok [4] by using theory the Green and Lindsay theory [5]. Chandersekharaish [6] established the micropolar thermoelasticity dependence on heat flux.

In the literature, there are three theories of thermoelasticity, namely, generalized theory, coupled theory and uncoupled theory. The classical uncoupled theory is based on two drawbacks. The first drawback corresponds to the propagation of wave with infinite speed in the uncoupled thermoelasticity theory and was studied by many researchers like Chandrasekhariah [7], Ferkas and Szekeres [8], Szekers [9]. The second drawback corresponds to the propagation of thermal wave with infinite speed. Biot [10] proposed the theory of coupled thermoelasticity to eradicate the other drawback. Hetnarski and Ignaczak [11] investigated many generalizations of the coupled theory and achieved a number of significant results. Concerning the GreenLindsay theory (GL), Lord-Shulman theory (LS) [12] and coupled theory (CT). Lord-Shulman determined the theory of generalized thermoelasticity to replace Fourier law by a new law named Maxwell-Catteneo law with one relaxation time. The emphasized theory confirmed the finite speed of propagation of elastic waves and heat waves when heat equations are converted into wave equations. The basic equations and relations remained same in this theory. In the Green-Lindsay theory is also recognized as the thermoelasticity theory depend on temperature rate or thermoelasticity theory with two relaxation times. In this theory, the equations for entropy and stress tensor are discussed with two relaxation times. Low temperature thermoelasticity theory is also

\footnotetext{
*-Corresponding author
} 
called the coupled theory studied by Ignaczak and Hetnarski [13]. This representation is described by a system of non-linear field equations. The thermoelasticity theory without energy dissipation in the third generalization of the coupled theory was proposed by Green and Naghdi [14] in which the classical Fourier law replaced the heat flux rate temperature gradient.

The problems of reflection and refraction of set of two coupled transverse waves and a set of two coupled longitudinal waves at a plane interface dividing a given medium into two half-spaces which are useful in engineering and geophysics areas. Many problems related to reflection and refraction of waves for a plane split into two media have been discussed by Tomar and Khurana [15]-[17], Tomar and Gogna [18, 19], Parfitt and Eringen [20], Tomar [21], Singh et al. [22], Zhang and coworkers [23]-[25], Achenbach [26], Sarkar and Tomar [27], Sahrawat et al. [28] and Poonam et al. [29]. Sahrawat et al. [30] investigated the fundamental solution and plane wave propagation in nonlocal couple stress micropolar thermoelastic solid without energy dissipation.

The main aim of the paper is to study the effect of three theories of thermoelasticity, namely, GreenLindsay theory (GL), Lord- Shulman theory (LS), Coupled theory (CT) on the propagation of a set of two coupled transverse waves and a set of two coupled longitudinal waves in a couple stress micropolar thermoviscous elastic solid medium. We study the reflection and refraction phenomenon of wave propagation at an interface of two couple stress microplolar thermoviscous elastic solid half spaces. It is found that there exist five waves, namely, longitudinal microrotational wave, a set of two coupled transverse waves and a set of two coupled longitudinal waves travelling with different speeds. The inequality of Energy ratios and reflection-refraction coefficients with respect to angle of incidence in both the half spaces has been studied numerically and graphically under the three theories of thermoelasticity, that is, LS, GL, CT.

\section{Basic relations and equations}

Following Tomar et al. [31], the Eqs for a couple stress micropolar thermoviscous elasticity are given by

$$
\begin{aligned}
& t_{i j}=\lambda^{\prime} \boldsymbol{u}_{r, r} \delta_{i j}+K^{\prime} e_{i j r} \boldsymbol{\phi}_{r}-\vartheta \theta \delta_{i j}-\vartheta t_{l} \delta_{k 2} \dot{\theta} \delta_{i j}+K^{\prime *} e_{i j r} \dot{\boldsymbol{\phi}}_{r}+\lambda^{* *} \dot{\boldsymbol{u}}_{r, r} \delta_{i j}+\left(\mu^{\prime}+K^{\prime}\right) \boldsymbol{u}_{j, i}+ \\
& +\left(\mu^{* *}+K^{* *}\right) \dot{\boldsymbol{u}}_{j, i}+\mu^{\prime} \boldsymbol{u}_{i, j}+\mu^{* *} \dot{\boldsymbol{u}}_{i, j} \\
& \boldsymbol{m}_{i j}=\alpha^{\prime} \boldsymbol{\phi}_{r, r} \delta_{i j}+\alpha^{\prime *} \dot{\phi}_{r, r} \delta_{i j}+\beta^{\prime} \phi_{i, j}+\beta^{\prime *} \dot{\phi}_{i, j}+\gamma^{\prime} \phi_{j, i}+\gamma^{* *} \dot{\phi}_{j, i} \\
& \rho_{0} \eta=\vartheta u_{r, r} \delta_{i j}+a \\
& Y_{i}+t_{0} \delta_{k l} \dot{\theta}=K_{t} \theta_{, i}
\end{aligned}
$$

where $\boldsymbol{u}$ denotes the displacement vector; $\boldsymbol{\phi}$ denotes the microrotation vector; Dot (.) represents differentiation with respect to time; comma (, ) represents the spatial derivative; $\nabla^{2}$ denotes the Laplacian operator; $\lambda^{\prime}$ and $\mu^{\prime}$ are Lame's constants; $K^{\prime}, \alpha^{\prime}, \beta^{\prime}, \gamma^{\prime}, \lambda^{* *}, \mu^{* *}, \alpha^{* *}, \beta^{\prime *}, \gamma^{* *}, \vartheta, K_{t}$ and $a$ are material constants; $t_{i j}$ represents the stress tensor; $m_{i j}$ represents the couple stress tensor; $\eta$ denotes entropy per unit mass; $\rho_{0}$ denotes density of the medium; $Y_{i}$ is the heat flux vector; $\theta$ denotes the change in temperature; $T_{0}$ is the ambient temperature and $\delta_{i j}$ denotes Kronecker's delta. Here $t_{0}$ and $t_{1}$ are the relaxation times and $k$ identifies the different theories of thermoelasticity. The case $k=1$ corresponds to the LST and the case $k=2$ corresponds to the GLT. When both $t_{0}$ and $t_{1}$ vanish, it corresponds to the CT of thermoelasticity.

Now, we introduce the following dimensionless quantities: 


$$
x_{i}^{\prime}=\frac{x_{i}}{l_{0}}, \quad u_{i}^{\prime}=\frac{u_{i}}{l_{0}}, \quad \phi_{i}^{\prime}=\frac{\phi_{i}}{l_{0}}, \quad t^{\prime}=\frac{c_{0} t}{l_{0}}, \quad T^{\prime}=\frac{\theta}{T_{0}}, \quad t_{i j}^{\prime}=\frac{t_{i j}}{l_{0} \vartheta T_{0}}, \quad m_{i j}^{\prime}=\frac{m_{i j}}{l_{0} \vartheta T_{0}}
$$

where $c_{0}$ and $l_{0}$ are the standard velocity and standard displacement, respectively.

With the help of these non-dimensional quantities, Eqs (2.1)-(2.4) become

$$
\begin{aligned}
& \left(\lambda_{2}+\mu_{2}\right) \nabla(\nabla \cdot \boldsymbol{u})+\left(\lambda_{2}^{*}+\mu_{2}^{*}\right) \nabla(\nabla \cdot \dot{\boldsymbol{u}})+\left(\mu_{2}+K_{2}\right) \nabla^{2} \boldsymbol{u}+ \\
& +\left(\mu_{2}^{*}+K_{2}^{*}\right) \nabla^{2} \dot{\boldsymbol{u}}+K_{2} \nabla \times \phi+K_{2}^{*} \nabla \times \dot{\boldsymbol{\phi}}-\vartheta_{1} T_{, i}-\vartheta_{2} \dot{T}_{, i}=\rho \ddot{\boldsymbol{u}}, \\
& \left(\alpha_{2}+\beta_{2}\right) \nabla(\nabla \cdot \boldsymbol{\phi})+\left(\alpha_{2}^{*}+\beta_{2}^{*}\right) \nabla(\nabla \cdot \boldsymbol{\phi})+\gamma_{2} \nabla^{2} \boldsymbol{\phi}+\gamma_{2}^{*} \nabla^{2} \dot{\boldsymbol{\phi}}+ \\
& +K_{2} \nabla \times \boldsymbol{u}+K_{2}^{*} \nabla \times \dot{\boldsymbol{u}}-2 K_{2}^{*} \dot{\boldsymbol{\phi}}-2 K_{2} \phi=r_{l} \ddot{\boldsymbol{\phi}}, \\
& \left(1+n_{l} \boldsymbol{\delta}_{l k} \frac{\partial}{\partial t}\right) \dot{\boldsymbol{T}}+\left(\frac{\vartheta_{1}}{\tau_{3}}+\frac{\vartheta_{1}}{\tau_{2}} \delta_{l k}\right) \nabla \cdot \dot{\boldsymbol{u}}=k_{1} \nabla^{2} \boldsymbol{T}
\end{aligned}
$$

where

$$
\begin{aligned}
& \lambda_{1}=\lambda^{\prime}+\lambda^{* *} \frac{\partial}{\partial t}, \quad \mu_{1}=\mu^{\prime}+\mu^{* *} \frac{\partial}{\partial t}, \quad K_{1}=K^{\prime}+K^{* *} \frac{\partial}{\partial t}, \quad \alpha_{1}=\alpha^{\prime}+\alpha^{*} \frac{\partial}{\partial t}, \\
& \beta_{1}=\beta^{\prime}+\beta^{* *} \frac{\partial}{\partial t}, \quad \gamma_{1}=\gamma^{\prime}+\gamma^{*} \frac{\partial}{\partial t}, \quad \lambda_{2}=\frac{\lambda_{1}}{\rho_{0} c_{0}^{2}}, \quad \lambda_{2}^{*}=\frac{\lambda_{1}^{*}}{\rho_{0} c_{0} l_{0}}, \quad \mu_{2}=\frac{\mu_{1}}{\rho_{0} c_{0}^{2}}, \\
& \mu_{2}^{*}=\frac{\mu_{1}^{*}}{\rho_{0} c_{0} l_{0}}, \quad K_{2}=\frac{K_{1}}{\rho_{0} c_{0}^{2}}, \quad K_{2}^{*}=\frac{K_{1}^{*}}{\rho_{0} c_{0} l_{0}}, \quad \vartheta_{1}=\frac{\vartheta T_{0}}{\rho_{0} c_{0}^{2}}, \quad \vartheta_{2}=\frac{\vartheta T_{0} t_{1}}{\rho_{0} c_{0} l_{0}}, \\
& k_{1}=\frac{k_{t}}{c c_{0} l_{0}}, \quad n_{1}=\frac{t_{0} c_{0}}{l_{0}}, \quad r_{1}=\frac{j}{l_{0}^{2}}, \quad \tau_{2}=\frac{T_{0} l_{0} c}{\rho_{0} c_{0} l_{0}^{3}}, \quad \tau_{3}=\frac{T_{0} c}{\rho_{0} c_{0}^{2}}, \quad \alpha_{2}=\frac{\alpha_{1}}{\rho_{0} c_{0}^{2}}, \\
& \alpha_{2}^{*}=\frac{\alpha_{1}^{*}}{\rho_{0} c_{0} l_{0}}, \quad \beta_{2}=\frac{\beta_{1}}{\rho_{0} c_{0}^{2} l_{0}^{2}}, \quad \beta_{2}^{*}=\frac{\beta_{1}^{*}}{\rho_{0} c_{0} l_{0}^{3}}, \quad \gamma_{2}=\frac{\gamma_{1}}{\rho_{0} c_{0}^{2}}, \quad \gamma_{2}^{*}=\frac{\gamma_{1}^{*}}{\rho_{0} l_{0} c_{0}}, \\
& c_{1}^{\prime 2}=\left(\vartheta_{1}+\vartheta_{2} \delta_{2 k} \frac{\partial}{\partial t}\right), \quad c_{3}^{\prime 2}=\left(\frac{\vartheta_{1}}{\tau_{3}}+\frac{\vartheta_{1} \delta_{l k}}{\tau_{2}} \frac{\partial}{\partial t}\right), \quad c_{4}^{\prime 2}=\left(1+n_{l} \delta_{1 k} \frac{\partial}{\partial t}\right) .
\end{aligned}
$$

\section{Wave propagation}

By the Helmholtz decomposition theorem on vectors, we introduce a scalar potential $(\sigma, \xi)$ and a vector potential $(\boldsymbol{U}, \boldsymbol{\Pi})$, given by

$$
\boldsymbol{u}=\nabla \sigma+\nabla \times \boldsymbol{U}, \quad \nabla \cdot \boldsymbol{U}=0, \quad \phi=\nabla \xi+\nabla \times \boldsymbol{\Pi}, \quad \nabla \cdot \boldsymbol{\Pi}=0 .
$$

In the absence of body couples, body forces and heat equation, the Eqs of motion are obtained by using Eq.(3.1) in Eqs (2.5)-(2.7) as 


$$
\begin{aligned}
& -c_{1}^{\prime 2} T+\left(\lambda_{3}+2 \mu_{3}+K_{3}\right) \nabla^{2} \sigma-\ddot{\sigma}=0, \\
& \left(\mu_{3}+K_{3}\right) \nabla^{2} \mathbf{U}+K_{3} \nabla \times \boldsymbol{\Pi}-\ddot{\boldsymbol{U}}=0, \\
& \left(\alpha_{3}+\beta_{3}+\gamma_{3}\right) \nabla^{2} \xi-2 K_{3} \xi-\ddot{\xi}=0, \\
& \gamma_{3} \nabla^{2} \boldsymbol{\Pi}+K_{3} \nabla \times \boldsymbol{U}-2 K_{3} \boldsymbol{\Pi}-\ddot{\boldsymbol{\Pi}}=0, \\
& k_{l} \nabla^{2} T-c_{3}^{\prime 2} \nabla^{2} \dot{\sigma}-c_{4}^{\prime 2} \dot{T}=0
\end{aligned}
$$

where

$$
\begin{aligned}
& \lambda_{3}=\lambda_{2}+\lambda_{2}^{*} \frac{\partial}{\partial t}, \quad \mu_{3}=\mu_{2}+\mu_{2}^{*} \frac{\partial}{\partial t}, \quad K_{3}=K_{2}+K_{2}^{*} \frac{\partial}{\partial t}, \quad \alpha_{3}=\frac{1}{r} \alpha_{2}+\alpha_{2} \frac{\partial}{\partial t}, \\
& \beta_{3}=\frac{1}{r} \beta_{2}+\beta_{2}^{*} \frac{\partial}{\partial t}, \quad \gamma_{3}=\frac{1}{r} \gamma_{2}+\gamma_{2}^{*} \frac{\partial}{\partial t}, \quad c_{1}^{\prime 2}=\left(\vartheta_{1}+\vartheta_{2} \delta_{2 k} \frac{\partial}{\partial t}\right), \\
& c_{3}^{\prime 2}=\left(\frac{\vartheta_{1}}{\tau_{3}}+\frac{\vartheta_{1} \delta_{l k}}{\tau_{2}} \frac{\partial}{\partial t}\right), \quad c_{4}^{\prime 2}=\left(1+\mathrm{n}_{1} \delta_{l \mathrm{k}} \frac{\partial}{\partial \mathrm{t}}\right) .
\end{aligned}
$$

Eq.(3.2) and Eq.(3.6) show that $\sigma$ and $T$ are coupled; Eq.(3.3) and Eq.(3.5) show that $\boldsymbol{U}$ and $\boldsymbol{\Pi}$ are coupled; But Eq.(3.4) shows $\xi$ is uncoupled. The propagation of plane waves for Eqs (3.2)-(3.6) in the linear couple stress thermoviscous micropolar elasticity are given by

$$
\{\sigma, \xi, \boldsymbol{U}, \boldsymbol{\Pi}, T\}=\left\{a_{1}, b_{1}, \boldsymbol{A}, \boldsymbol{B}, c_{1}\right\} \exp \{l l(\boldsymbol{n} . \boldsymbol{r}-\mathrm{St})\},
$$

travelling with speed $S$ in Eqs (2.5)-(2.7), where $a_{1}, b_{1}, c_{1}, \boldsymbol{A}, \boldsymbol{B}$ are scalars, ' 1 ' denotes the iota, $\boldsymbol{n}$ is the unit vector and $\boldsymbol{r}$ is position vector. Putting the value of $\sigma$ and $T$ from Eq.(3.7) in Eq.(3.2) and Eq.(3.6), we will obtain

$$
X_{1} S^{4}+X_{2} S^{2}+X_{3}=0
$$

The roots of the Eq. (3.8) are given by

$$
S_{1,2}^{2}=\frac{-Y_{2} \pm \sqrt{Y_{2}^{2}-4 Y_{1} Y_{3}}}{2 Y_{1}}
$$

where $Y_{s}(s=1,2,3)$ are provided in Appendix $A_{1}$.

From Eq.(3.2), we will obtain the relation between $a_{1}$ and $\mathrm{c}_{1}$

$$
a_{1}=\frac{-c_{1}^{\prime 2}}{\omega^{2}-l^{2}\left(\zeta_{3}-1 \omega \zeta_{3}^{*}\right)} c_{l}
$$

where $\zeta_{3}$ and $\zeta_{3}^{*}$ are provided in Appendix $A_{1}$. 
Using Eq.(3.7) in Eq.(3.1), we will obtain the displacement vector as follows

$$
\boldsymbol{u}=\mathfrak{l l a} a_{1} \boldsymbol{\operatorname { e x p }}\{\mathrm{l} l(\boldsymbol{n} . \boldsymbol{r}-\mathrm{St})\},
$$

which explains that the directions of $\boldsymbol{u}$ and $\boldsymbol{n}$ are same. The directions of the displacement of wave and travelling of wave are same, so this is longitudinal in nature and known as longitudinal displacement wave. Also, the direction of wave related to $T$ is longitudinal in nature (using Eq.(3.10) and Eq.(3.11)) and known as longitudinal thermal wave. Since, both the waves are coupled and hence, known as "coupled longitudinal waves".

Putting the value of $\xi$ from Eq. (3.7) into Eq. (3.4), we will obtain the longitudinal microrotational wave

$$
l^{2}=\frac{\omega^{2}-2 \zeta_{2}}{C}
$$

where $C$ and $\zeta_{2}$ are provided in Appendix $A_{1}$.

Using Eq.(3.7) in Eq.(3.3) and Eq.(3.5), we will obtain a quadratic equation in terms of $S^{2}$ such that

$$
X_{1} S^{4}+X_{2} S^{2}+X_{3}=0
$$

The roots of the Eq.(3.13) are given by

$$
S_{3,4}^{2}=\frac{-X_{2} \pm \sqrt{X_{2}^{2}-4 X_{1} X_{3}}}{2 X_{1}}
$$

where $X_{s}(\mathrm{~s}=1,2,3)$ are provided in Appendix $A_{l}$.

Using Eq.(3.7) in Eq.(3.1), we will obtain

$$
\begin{aligned}
& \boldsymbol{n} \cdot \boldsymbol{A}=\boldsymbol{n} \cdot \boldsymbol{B}, \\
& \boldsymbol{A}=\frac{-1 l \zeta_{2}}{\left[l^{2}\left(\zeta_{1}-1 \omega \zeta_{1}^{*}\right)-\omega^{2}\right]} \boldsymbol{B}
\end{aligned}
$$

where $\boldsymbol{A}$ and $\boldsymbol{B}$ lie in the same plane and mutually perpendicular to each other. From Eq.(3.16), we observe that if $\boldsymbol{A} \equiv 0$, then $\boldsymbol{B} \equiv 0$, making both $\boldsymbol{U}$ and $\boldsymbol{\Pi}$ vanish. These two waves vanish together. Eq.(3.1) shows that $\boldsymbol{U}$ and $\boldsymbol{I}$ are normal to each other and also both are normal to the direction of propagation $\boldsymbol{n}$ and known as transverse waves. Since both the waves are coupled in nature and hence, known as "coupled transverse waves". $\zeta_{2}, \zeta_{1}$ and $\zeta_{1}^{*}$ are provided in Appendix $A_{1}$.

\section{Model}

We consider two distinct couple stress micropolar thermoviscous elastic half-spaces, namely, $M_{1}$ and $M_{2}$, respectively, in perfect contact in the Cartesian co-ordinate system $O x_{1} x_{2} x_{3}$ in which the plane interface coincides with $x_{1} x_{3}$ - plane. $M_{1}$ and $M_{2}$ occupy the region $x_{3} \geq 0$ and $x_{3} \leq 0$, respectively, as shown in Fig.1. The elastic moduli corresponding to region $M_{1}$ are $\lambda_{2}, \mu_{2}, K_{2}, \alpha_{2}, \beta_{2}, \gamma_{2}^{*}, \lambda_{2}^{*}, \mu_{2}^{*}, K_{2}^{*}, \alpha_{2}^{*}, \beta_{2}^{*}, \gamma_{2}^{*}$ those corresponding to region $M_{2}$ are $\lambda_{2}^{\prime}, \mu_{2}^{\prime}, K_{2}^{\prime}, \alpha_{2}^{\prime}, \beta_{2}^{\prime}, \gamma_{2}^{\prime *}, \lambda_{2}^{\prime *}, \mu_{2}^{\prime *}, K_{2}^{\prime *}, \alpha_{2}^{\prime *}, \beta_{2}^{\prime *}, \gamma_{2}^{\prime *}$. 


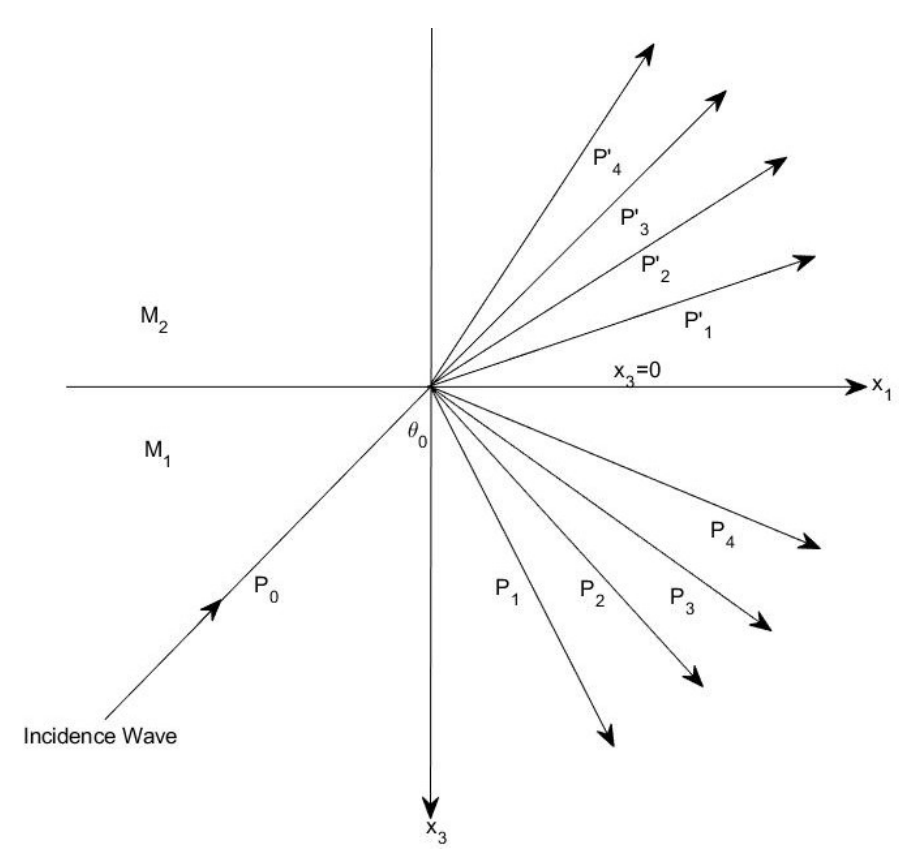

Fig.1. The geometry of the problem.

\section{Boundary conditions}

Solving Eqs (2.1)-(2.4) and Eq.(3.1), we will obtain the components of stress tensor, couple stress tensor, displacement and microrotation for mediums $M_{1}$ and $M_{2}$ are given by

$$
\begin{aligned}
& u_{1}=\sigma_{, 1}-U_{2,3}, \quad u_{3}=\sigma_{, 3}+U_{2,1}, \quad \phi_{2}=\Pi_{1,3}-\Pi_{3,1}, \\
& t_{33}=-C_{1}^{\prime 2} T+\lambda_{3} \sigma_{, 11}+\left(\lambda_{3}+2 \mu_{3}+K_{3}\right) \sigma_{, 33}+\left(2 \mu_{3}+K_{3}\right) U_{2,13}, \\
& t_{13}=\left(2 \mu_{3}+K_{3}\right) \sigma_{, 13}+\mu_{3} U_{2,11}-\left(\mu_{3}+K_{3}\right) U_{2,33} k_{1} \phi_{2}, \quad q_{k}=k_{1} T_{, k}, \quad m_{32}=\gamma_{3} \phi_{2,3}, \\
& u_{1}^{\prime}=\sigma_{, 1}^{\prime}-U_{2,3}^{\prime}, \quad u_{3}^{\prime}=\sigma_{, 3}^{\prime}+U_{2,1}^{\prime}, \quad \phi_{2}^{\prime}=\Pi_{1,3}^{\prime}-\Pi_{3,1}^{\prime}, \\
& t_{33}^{\prime}=-C_{1}^{\prime 2} T^{\prime}+\lambda_{3}^{\prime} \sigma_{, 11}^{\prime}+\left(\lambda_{3}^{\prime}+2 \mu_{3}^{\prime}+K_{3}^{\prime}\right) \sigma_{, 33}^{\prime}+\left(2 \mu_{3}^{\prime}+K_{3}^{\prime}\right) U_{2,13}^{\prime}, \\
& t_{13}^{\prime}=\left(2 \mu_{3}^{\prime}+K_{3}^{\prime}\right) \sigma_{, 13}^{\prime}+\mu_{3}^{\prime} U_{2,11}^{\prime}-\left(\mu_{3}^{\prime}+K_{3}^{\prime}\right) U_{2,33}^{\prime} k_{1}^{\prime} \phi_{2}^{\prime}, \\
& q_{k}^{\prime}=k_{1}^{\prime} T_{, k}^{\prime}, \quad m_{32}^{\prime}=\gamma_{3}^{\prime} \phi_{2,3}^{\prime} .
\end{aligned}
$$

The boundary conditions at the interface of two half spaces are given by

$$
\begin{aligned}
& t_{33}=t_{33}^{\prime}, \quad t_{31}=t_{31}^{\prime}, \quad m_{32}=m_{32}^{\prime}, \quad q_{k}=q_{k}^{\prime}, \\
& u_{1}=u_{1}^{\prime}, \quad u_{3}=u_{3}^{\prime}, \quad \phi_{2}=\phi_{2}^{\prime}, \quad T=T^{\prime} .
\end{aligned}
$$




\section{Incidence of coupled longitudinal waves}

\subsection{Incidence of longitudinal displacement wave}

At the interface $x_{3}=0$, let us consider a longitudinal wave of amplitude $P_{0}$ with speed $S_{1}$, propagating through the half space $M_{l}$ with wave number $l_{l}$ and making an angle $\theta_{0}$.

The boundary conditions for the problem are given by:

Reflected waves in medium $M_{1}$ : A set of two coupled longitudinal waves and a set of two coupled transverse waves with amplitude $P_{1}, P_{2}, P_{3}$ and $P_{4}$ propagating with speeds $S_{1}, S_{2}, S_{3}$ and $S_{4}$ making an angle $\theta_{1}, \theta_{2}, \theta_{3}$ and $\theta_{4}$ respectively with the normal.

Reflected waves in medium $M_{2}$ : A set of two coupled longitudinal waves and a set of two coupled transverse waves with amplitude $P_{1}^{\prime}, P_{2}^{\prime}, P_{3}^{\prime}$ and $P_{4}^{\prime}$ propagating with speeds $S_{1}^{\prime}, S_{2}^{\prime}, S_{3}^{\prime}$ and $S_{4}^{\prime}$ making an angle $\theta_{1}^{\prime}, \theta_{2}^{\prime}, \theta_{3}^{\prime}$ and $\theta_{4}^{\prime}$ respectively with the normal.

Equations (3.9) and Eq. (3.14) show the speeds $S_{1}, S_{2}, S_{3}$ and $S_{4}$, respectively. The equations for speeds $S_{1}^{\prime}, S_{2}^{\prime}, S_{3}^{\prime}$ and $S_{4}^{\prime}$ are same as for $S_{1}, S_{2}, S_{3}$ and $S_{4}$, respectively, with the primes at significant places. The total wave field is given by (Tomar et al. [19])

$$
\begin{aligned}
& \sigma=P_{0} \exp \left\{l l_{l}\left(\sin \theta_{0} x_{1}-\cos \theta_{0} x_{3}\right)-\imath \omega_{1} t\right\}+ \\
& +\sum_{r=1,2} \mathrm{P}_{\mathrm{r}} \exp \left\{l l_{r}\left(\sin \theta_{r} x_{1}+\cos \theta_{r} x_{3}\right)-1 \omega_{r} t\right\} \text {, } \\
& T=\varkappa_{1} P_{0} \exp \left\{l_{l}\left(\sin \theta_{0} x_{1}-\cos \theta_{0} x_{3}\right)-1 \omega_{1} t\right\}+ \\
& +\sum_{r=1,2} \varkappa_{r} \mathrm{P}_{\mathrm{r}} \exp \left\{1 l_{r}\left(\sin \theta_{r} x_{1}+\cos \theta_{r} x_{3}\right)-1 \omega_{r} t\right\}, \\
& U=\sum_{r=3,4} \mathrm{P}_{\mathrm{r}} \exp \left\{l_{r}\left(\sin \theta_{r} x_{1}+\cos \theta_{r} x_{3}\right)-1 \omega_{r} t\right\}, \\
& \Pi=\sum_{r=3,4} \eta_{r} \mathrm{P}_{\mathrm{r}} \exp \left\{l_{r}\left(\sin \theta_{r} x_{1}+\cos \theta_{r} x_{3}\right)-1 \omega_{r} t\right\}, \\
& \sigma^{\prime}=\sum_{r=1,2} \mathrm{P}_{\mathrm{r}}^{\prime} \exp \left\{\mathrm{l} l_{r}\left(\sin \theta_{r} x_{1}+\cos \theta_{r} x_{3}\right)-1 \omega_{r} t\right\}, \\
& T^{\prime}=\sum_{r=1,2} \varkappa_{r}^{\prime} \mathrm{P}_{\mathrm{r}}^{\prime} \exp \left\{l l_{r}\left(\sin \theta_{r} x_{1}+\cos \theta_{r} x_{3}\right)-\imath \omega_{r} t\right\}, \\
& \boldsymbol{U}^{\prime}=\sum_{r=3,4} \mathrm{P}_{\mathrm{r}}^{\prime} \exp \left\{l l_{r}\left(\sin \theta_{r} x_{1}+\cos \theta_{r} x_{3}\right)-\imath \omega_{r} t\right\}, \\
& \boldsymbol{\Pi}^{\prime}=\sum_{r=3,4} \eta_{r}^{\prime} \mathrm{P}_{\mathrm{r}}^{\prime} \exp \left\{l_{r}\left(\sin \theta_{r} x_{1}+\cos \theta_{r} x_{3}\right)-1 \omega_{r} t\right\}
\end{aligned}
$$


where $\left(l_{q}, l_{q}^{\prime}\right)$ and $\left(S_{q}, S_{q}^{\prime}\right)$ are the wave numbers and phase speeds corresponding media $M_{1}$ and $M_{2}$, respectively. $\left(\omega_{l}=l_{l} S_{l}, \omega_{l}^{\prime}=l_{l}^{\prime} S_{l}^{\prime}\right)$ denotes the angular frequencies of the mediums $M_{1}$ and $M_{2}$, respectively. $\varkappa_{1,2}$ and $\eta_{3,4}$ are the coupling parameters obtained by the Eq.(3.11) and Eq.(3.16) respectively

$$
\varkappa_{1,2}=\frac{-c_{1}^{\prime 2}}{\omega^{2}-l^{2}\left(\zeta_{3}-1 \omega \zeta_{3}^{*}\right)}, \quad \eta_{3,4}=\frac{-1 l \zeta_{2}}{\left[l^{2}\left(\zeta_{1}-1 \omega \zeta_{1}^{*}\right)-\omega^{2}\right]} .
$$

Using Eq. (5.1) in Eqs (6.1) -(6.8), we will obtain

$$
\begin{aligned}
& {\left[\zeta_{4}+\zeta_{5} \cos ^{2} \theta_{0}+\frac{\nabla_{4} \varkappa_{1}}{l_{1}^{2}}\right] l_{1}^{2} P_{0}+\sum_{r=1,2}\left[\zeta_{4}^{\prime}+\zeta_{5}^{\prime} \cos ^{2} \theta_{r}+\frac{\nabla_{4} \varkappa_{r}}{l_{r}^{2}}\right] l_{r}^{2} \mathrm{P}_{\mathrm{r}}+} \\
& +\sum_{r=3,4} \zeta_{5} \sin \theta_{r} \cos \theta_{r} l_{r}^{2} \mathrm{P}_{\mathrm{r}}-\sum_{r=1,2}\left[\zeta_{4}^{\prime}+\zeta_{5}^{\prime} \cos ^{2} \theta_{r}^{\prime}+\frac{\nabla_{4}^{\prime} \varkappa_{r}^{\prime}}{l_{r}^{\prime 2}}\right] l_{r}^{2} \mathrm{P}_{\mathrm{r}}^{\prime}+ \\
& +\sum_{r=3,4} \zeta_{5}^{\prime} \sin \theta_{r}^{\prime} \cos \theta_{r}^{\prime} l_{r}^{2} \mathrm{P}_{\mathrm{r}}^{\prime}=0 \\
& \zeta_{5} \sin \theta_{0} \cos \theta_{0} l_{1}^{2} P_{0}-\sum_{r=1,2} \zeta_{5} \sin \theta_{r} \cos \theta_{r} l_{r}^{2} \mathrm{P}_{\mathrm{r}}+ \\
& +\sum_{3,4}\left[\zeta_{6} \cos 2 \theta_{r}+\zeta_{7} \cos ^{2} \theta_{r}-\frac{\zeta_{7} \eta_{r}}{l_{r}^{2}}\right] l_{r}^{2} P_{r}+ \\
& -\sum_{r=1,2} \zeta_{5}^{\prime} \sin \theta_{r}^{\prime} \cos \theta_{r}^{\prime} l_{r}^{2} \mathrm{P}_{\mathrm{r}}^{\prime}-\sum_{3,4}\left[\zeta_{6}^{\prime} \cos 2 \theta_{r}^{\prime}+\zeta_{7}^{\prime} \cos ^{2} \theta_{r}^{\prime}-\frac{\zeta_{7}^{\prime} \eta_{r}^{\prime}}{l_{r}^{\prime 2}}\right] l_{r}^{\prime 2} \mathrm{P}_{\mathrm{r}}^{\prime}=0 \text {, } \\
& \sum_{3,4} \zeta_{2}^{*} \eta_{r} \cos \theta_{r} l_{r} P_{r}+\sum_{3,4} \zeta_{2}^{* *} \eta_{r}^{\prime} \cos \theta_{r}^{\prime} l_{r}^{\prime} P_{r}^{\prime}=0 \\
& k_{1} \varkappa_{1} \cos \theta_{0} l_{1} P_{0}-\sum_{r=1,2} k_{1} \varkappa_{r} \cos \theta_{r} l_{r} \mathrm{P}_{\mathrm{r}}-\sum_{r=1,2} k_{1}^{\prime} \varkappa_{r}^{\prime} \cos \theta_{r}^{\prime} l_{r}^{\prime} \mathrm{P}_{\mathrm{r}}^{\prime}=0 \\
& \sin \theta_{0} l_{1} P_{0}+\sum_{r=1,2} \sin \theta_{r} l_{r} \mathrm{P}_{\mathrm{r}}-\sum_{r=3,4} \cos \theta_{r} l_{r} \mathrm{P}_{\mathrm{r}}-\sum_{r=1,2} \sin \theta_{r}^{\prime} l_{r}^{\prime} \mathrm{P}_{\mathrm{r}}^{\prime}-\sum_{r=3,4} \cos \theta_{r}^{\prime} l_{r}^{\prime} \mathrm{P}_{\mathrm{r}}^{\prime}=0 \\
& \cos \theta_{0} l_{1} P_{0}-\sum_{r=1,2} \cos \theta_{r} l_{r} \mathrm{P}_{\mathrm{r}}-\sum_{r=3,4} \sin \theta_{r} l_{r} \mathrm{P}_{r}-\sum_{r=1,2} \cos \theta_{r}^{\prime} l_{r}^{\prime} \mathrm{P}_{\mathrm{r}}^{\prime}+\sum_{r=3,4} \sin \theta_{r}^{\prime} l_{r}^{\prime} \mathrm{P}_{\mathrm{r}}^{\prime}=0, \\
& \eta_{3} P_{3}+\eta_{4} P_{4}-\eta_{3}^{\prime} P_{3}^{\prime}-\eta_{4}^{\prime} P_{4}^{\prime}=0 \\
& \varkappa_{1} P_{0}+\varkappa_{1} P_{1}+\varkappa_{2} P_{2}-\varkappa_{1}^{\prime} P_{1}^{\prime}-\varkappa_{2}^{\prime} P_{2}^{\prime}=0 .
\end{aligned}
$$


Equations (6.9)-(6.16) in the matrix form can be written as

$$
[H][Q]=[Z]
$$

where $[H]=\left[h_{i j}\right]$ is a $8 \times 8$ square matrix, $[Q]=\left[Q_{1}, Q_{2}, Q_{3}, Q_{4}, Q_{5}, Q_{6}, Q_{7}, Q_{8}\right]^{\prime}$ is a $8 \times 1$ column matrix, 'dash' denotes the transpose of the matrix. $Q_{r}=\frac{\mathrm{P}_{\mathrm{r}}}{P_{0}}(r=1,2,3,4)$ and $Q_{t}=\frac{P_{t-4}^{\prime}}{P_{0}}(t=5,6,7,8)$ are the reflection and refraction coefficients. All non-zero entries of the matrices $[H]$ and $[Z]$ are defined in Appendix $A_{1}$.

\subsection{Energy partitioning}

In this problem, we must show the energy balance to establish the reflection and refraction coefficients. The rate of transmission $\left\langle O^{*}\right\rangle$ per unit area is (Achenbach [26])

$$
O^{*}=t_{33} \dot{u}_{3}+t_{31} u_{1}+m_{32} \dot{\phi}_{2}
$$

The energy carried along the incident wave, reflected and refracted waves is denoted by $\left\langle O_{0}\right\rangle,\left\langle O_{i}\right\rangle$ and $\left\langle O_{i}^{\prime}\right\rangle$ respectively and the energy ratios $\left\langle E_{i}\right\rangle(i=1,2 \ldots .8)$ such that

$$
E_{i}=\frac{\left\langle O_{i}\right\rangle}{\left\langle O_{0}\right\rangle}
$$

The energy ratios for the reflected and refracted waves are given in Appendix $A_{1}$.

When the coupled longitudinal wave of amplitude $P_{0}$ with $S_{2}$ and $l_{2}$ as the speed and wave number propagating through medium $M_{1}$ by making angle $\theta_{0}$, then we follow the same process and obtain a matrix similar to Eq.(6.17), with the modified values given in Appendix $A_{2}$. The matrix [Q] and [Z] remains same. The expressions of Energy ratios are also given in Appendix $A_{2}$.

\section{Incidence of coupled transverse waves}

We examine a coupled transverse wave having amplitude $P_{3}$ travelling with speed $S_{3}$ for an angle $\theta_{0}$, at the interface of two half-spaces using boundary conditions as given in Eq.(5.1) The reflected and refracted waves obtained in incidence of a set of two coupled longitudinal waves are same.

The total wave field equations in Medium $M_{1}$ are given by (Tomar et al. [19])

$$
\begin{aligned}
& \sigma=\sum_{r=1,2} \mathrm{P}_{\mathrm{r}} \exp \left\{l_{r}\left(\sin \theta_{r} x_{1}+\cos \theta_{r} x_{3}\right)-1 \omega_{r} t\right\}, \\
& T=\sum_{r=1,2} \varkappa_{r} \mathrm{P}_{\mathrm{r}} \exp \left\{l l_{r}\left(\sin \theta_{r} x_{1}+\cos \theta_{r} x_{3}\right)-1 \omega_{r} t\right\},
\end{aligned}
$$




$$
\begin{aligned}
& U=P_{0} \exp \left\{l l_{3}\left(\sin \theta_{0} x_{1}+\cos \theta_{0} x_{3}\right)-1 \omega_{3} t\right\}+ \\
& +\sum_{q=3,4} P_{q x_{2}} e_{x_{2}} \exp \left\{l l_{q}\left(\sin \theta_{q} x_{1}-\cos \theta_{q} x_{3}\right)-1 \omega_{q} t\right\}, \\
& \Pi=\eta_{3} P_{0} \exp \left\{l l_{3}\left(\sin \theta_{0} x_{1}+\cos \theta_{0} x_{3}\right)-1 \omega_{3} t\right\}+ \\
& +\sum_{q=3,4}\left(Q_{q x_{1}} e_{x_{1}}+Q_{q x_{3}} e_{X_{3}}\right) \exp \left\{l_{q}\left(\sin \theta_{q} x_{1}-\cos \theta_{q} x_{3}\right)-1 \omega_{q} t\right\} .
\end{aligned}
$$

The total wave field equations in medium $M_{2}$ are same as given in the case of a longitudinal displacement wave. Using boundary conditions (5.1) in Eqs (6.5)-(6.8) and Eqs (7.1)-(7.4), we will obtain

$$
\begin{aligned}
& -\zeta_{5} \sin \theta_{0} \cos \theta_{0} l_{3}^{2} P_{0}+\sum_{r=1,2}\left[\zeta_{4}^{\prime}+\zeta_{5}^{\prime} \cos ^{2} \theta_{r}+\frac{\nabla_{4} \varkappa_{r}}{l_{r}^{2}}\right] l_{r}^{2} \mathrm{P}_{\mathrm{r}}+ \\
& +\sum_{r=3,4} \zeta_{5} \sin \theta_{r} \cos \theta_{r} l_{r}^{2} \mathrm{P}_{\mathrm{r}}-\sum_{r=1,2}\left[\zeta_{4}^{\prime}+\zeta_{5}^{\prime} \cos ^{2} \theta_{r}^{\prime}+\frac{\nabla_{4}^{\prime} \varkappa_{r}^{\prime}}{l_{r}^{\prime 2}}\right] l_{r}^{\prime 2} \mathrm{P}_{\mathrm{r}}^{\prime}+ \\
& +\sum_{r=3,4} \zeta_{5}^{\prime} \sin \theta_{r}^{\prime} \cos \theta_{r}^{\prime} l_{r}^{\prime 2} \mathrm{P}_{\mathrm{r}}^{\prime}=0 \\
& {\left[\zeta_{6} \cos 2 \theta_{r}+\zeta_{7} \cos ^{2} \theta_{r}-\frac{\zeta_{7} \eta_{r}}{l_{r}^{2}}\right] l_{3}^{2} P_{0}-\sum_{r=1,2} \zeta_{5} \sin \theta_{r} \cos \theta_{r} l_{r}^{2} \mathrm{P}_{\mathrm{r}}+} \\
& +\sum_{3,4}\left[\zeta_{6} \cos 2 \theta_{r}+\zeta_{7} \cos ^{2} \theta_{r}-\frac{\zeta_{7} \eta_{r}}{l_{r}^{2}}\right] l_{r}^{2} \mathrm{P}_{\mathrm{r}}-\sum_{r=1,2} \zeta_{5}^{\prime} \sin \theta_{r}^{\prime} \cos \theta_{r}^{\prime} l_{r}^{\prime 2} \mathrm{P}_{\mathrm{r}}^{\prime}+ \\
& -\sum_{3,4}\left[\zeta_{6}^{\prime} \cos 2 \theta_{r}^{\prime}+\zeta_{7}^{\prime} \cos ^{2} \theta_{r}^{\prime}-\frac{\zeta_{7}^{\prime} \eta_{r}^{\prime}}{l_{r}^{\prime 2}}\right] l_{r}^{2} \mathrm{P}_{\mathrm{r}}^{\prime}=0 \\
& \zeta_{2}^{*} \eta_{r} \cos \theta_{r} l_{r} \mathrm{P}_{\mathrm{r}}-\sum_{3,4} \zeta_{2}^{*} \eta_{r} \cos \theta_{r} l_{r} \mathrm{P}_{\mathrm{r}}+\sum_{3,4} \zeta_{2}^{* *} \eta_{r}^{\prime} \cos \theta_{r}^{\prime} l_{r}^{\prime} \mathrm{P}_{\mathrm{r}}^{\prime}=0 \\
& -\sum_{r=1,2} k_{1} \varkappa_{r} \cos \theta_{r} l_{r} \mathrm{P}_{\mathrm{r}}-\sum_{r=1,2} k_{1}^{\prime} \varkappa_{r}^{\prime} \cos \theta_{r}^{\prime} l_{r}^{\prime} \mathrm{P}_{\mathrm{r}}^{\prime}=0 \\
& \cos \theta_{0} l_{3} P_{0}+\sum_{r=1,2} \sin \theta_{r} l_{r} \mathrm{P}_{\mathrm{r}}-\sum_{r=3,4} \cos \theta_{r} l_{r} \mathrm{P}_{\mathrm{r}}-\sum_{r=1,2} \sin \theta_{r}^{\prime} l_{r}^{\prime} \mathrm{P}_{\mathrm{r}}^{\prime}-\sum_{r=3,4} \cos \theta_{r}^{\prime} l_{r}^{\prime} \mathrm{P}_{\mathrm{r}}^{\prime}=0, \\
& \sin \theta_{0} l_{3} P_{0}-\sum_{r=1,2} \cos \theta_{r} l_{r} \mathrm{P}_{r}-\sum_{r=3,4} \sin \theta_{r} l_{r} \mathrm{P}_{r}-\sum_{r=1,2} \cos \theta_{r}^{\prime} l_{r}^{\prime} \mathrm{P}_{\mathrm{r}}^{\prime}+\sum_{r=3,4} \sin \theta_{r}^{\prime} l_{r}^{\prime} \mathrm{P}_{\mathrm{r}}^{\prime}=0, \\
& \eta_{3} P_{0}+\eta_{3} P_{3}+\eta_{4} P_{4}-\eta_{3}^{\prime} P_{3}^{\prime}-\eta_{4}^{\prime} P_{4}^{\prime}=0,
\end{aligned}
$$




$$
\varkappa_{1} P_{1}+\varkappa_{2} P_{2}-\varkappa_{1}^{\prime} P_{1}^{\prime}-\varkappa_{2}^{\prime} P_{2}^{\prime}=0 .
$$

Equations (7.5)-(7.12) in the matrix form can be written as

$$
[U][V]=[W]
$$

where $[U]=\left[U_{i j}\right]$ is a $8 \times 8$ square matrix, $[V]=\left[V_{1}, V_{2}, V_{3}, V_{4}, V_{5}, V_{6}, V_{7}, V_{8}\right]^{\prime}$ is a $8 \times 1$ column matrix. $V_{r}=\frac{\mathrm{P}_{\mathrm{r}}}{P_{0}}(r=1,2,3,4)$ and $V_{t}=\frac{P_{t-4}^{\prime}}{P_{0}}(t=5,6,7,8)$ are the reflection and refraction coefficients. All non-zero entries of the matrices $[U]$ and $[W]$ are provided in Appendix $B_{1}$.

\subsection{Energy partitioning}

The logical expressions of the energy ratios $E_{i}(i=1,2 \ldots . .8)$ in the medium $M_{1}$ and $M_{2}$ for the coupled transverse waves are given in Appendix $B_{1}$. When the coupled transverse wave of amplitude $P_{4}$ with $S_{4}$ and $l_{4}$ as the speed and wave number propagating through medium $M_{1}$ by making angle $\theta_{0}$, then we follow the same process and obtain a matrix similar to (7.13), with the modified values given in Appendix $B_{2}$. The matrix $[\mathrm{V}]$ and $[\mathrm{W}]$ remains same. The expressions of Energy ratios are also given in Appendix $B_{2}$.

\section{Special cases}

In this section, the speeds of propagating plane waves are reduced by using the conditions of different theories of thermoviscous elasticity.

\subsection{Lord-Shulman theory}

If we take $k=1$, all the equations remain same except those which contain Kronecker's delta. Therefore, we have

$$
c_{1}^{\prime 2}=\vartheta_{1}, \quad c_{3}^{\prime 2}=\left(\frac{\vartheta_{1}}{\tau_{3}}+\frac{\vartheta_{1}}{\tau_{2}} \frac{\partial}{\partial t}\right), \quad c_{4}^{\prime 2}=\left(1+n_{1} \frac{\partial}{\partial t}\right) .
$$

Using these expressions, we can find the speeds of various waves in terms of the LS theory.

\subsection{Green-Lindsay theory}

If we put $k=2$, all the terms which contain Kronecker's delta can be reduced in the form

$$
c_{1}^{\prime 2}=\left(\vartheta_{1}+\vartheta_{2} \frac{\partial}{\partial t}\right), \quad c_{3}^{\prime 2}=\frac{\vartheta_{1}}{\tau_{3}}, \quad c_{4}^{\prime 2}=1
$$

Using these expressions, we can find the speed of various waves in terms of GL theory.

\subsection{Coupled theory}

When we take $t_{0}=t_{1}=0$, then the expressions which contains the terms $t_{0}, t_{1}$ become 


$$
c_{1}^{\prime 2}=\left(\vartheta_{1}+\vartheta_{2} \frac{\partial}{\partial t}\right), \quad c_{3}^{\prime 2}=\frac{\vartheta_{1}}{\tau_{3}}, \quad c_{4}^{\prime 2}=1
$$

Now, the equations of the given waves are reduced into classical elastic medium.

\section{Numerical analysis}

The reflection and refraction coefficients with their energy ratios and also with a set of two couple longitudinal wave and a set of two coupled transverse wave under three theories of thermoelasticity are calculated and drawn graphically using numerical values of different parameters from Kumar et al. [32] specified in Tab.1 and Tab.2 for both medium $M_{1}$ and $M_{2}$ with phase speeds $S_{r}$ and $S_{r}^{\prime} \quad(r=1,2,3,4)$ respectively by using MATLAB software.

Table 1. Numerical values of parameters.

\begin{tabular}{cccl}
\hline symbol & value & symbol & value \\
\hline$\lambda_{2}$ & $9.4 \times 10^{10} \mathrm{Nm}^{-2}$ & $\mu_{2}$ & $4.0 \times 10^{10} \mathrm{Nm}^{-2}$ \\
$K_{2}$ & $1.0 \times 10^{10} \mathrm{Nm}^{-2}$ & $\rho_{0}$ & $1.73 \times 10^{3} \mathrm{Kgm}^{-3}$ \\
$j$ & $0.2 \times 10^{-19} \mathrm{~m}^{2}$ & $\gamma_{2}$ & $0.779 \times 10^{-9} \mathrm{~N}$ \\
$\alpha_{2}$ & $2.33 \times 10^{-5} \mathrm{~N}$ & $\beta_{2}$ & $2.48 \times 10^{-5} \mathrm{~N}$ \\
$\vartheta$ & $0.9 \times 10^{-17} \mathrm{~N}$ & $K_{t}$ & $1.7 \times 10^{6} \mathrm{jKm}^{-1} \mathrm{~s}^{-1}$ \\
$t_{0}$ & 1.03 & $t_{1}$ & 1.04 \\
$\lambda_{1}^{*}$ & $0.5 \times 10^{1} 0 \mathrm{Nm}^{-2}$ & $\mu_{1}^{*}$ & $0.1 \times 10^{9} \mathrm{Nm}^{-2}$ \\
$\alpha_{1}^{*}$ & $5.27 \times 10^{3} \mathrm{~N}$ & $\beta_{1}^{*}$ & $3.17 \times 10^{-3} \mathrm{~N}^{*}$ \\
$\gamma_{1}^{*}$ & $0.5 \times 10^{3} \mathrm{~N}$ & $K_{1}^{*}$ & $0.5 \times 10^{10} \mathrm{~N}$ \\
$T_{0}$ & $0.293 \times 10^{3} \mathrm{~K}$ & $c$ & $1.04 \times 10^{3} \mathrm{JK}^{-1} \mathrm{~K}^{-1}$ \\
\hline
\end{tabular}

Table 2. Numerical values of parameters.

\begin{tabular}{|c|c|c|c|}
\hline symbol & value & symbol & value \\
\hline$\lambda_{1}^{\prime}$ & $75900 \times 10^{6} \mathrm{Nm}^{-2}$ & $\mu_{1}^{\prime}$ & $13500 \times 10^{6} \mathrm{Nm}^{-2}$ \\
\hline$K_{1}^{\prime}$ & $149 \times 10^{6} \mathrm{Nm}^{-2}$ & $\rho_{0}$ & $2.65 \times 10^{3} \mathrm{Kgm}^{-3}$ \\
\hline$j$ & $0.00000196 \mathrm{~m}^{2}$ & $\gamma_{1}^{\prime}$ & $0.0268 \times 10^{6} \mathrm{~N}$ \\
\hline$\alpha_{1}^{\prime}$ & $0.01 \times 10^{6} \mathrm{~N}$ & $\beta_{1}^{\prime}$ & $0.015 \times 10^{6} \mathrm{~N}$ \\
\hline$\vartheta$ & $0.9 \times 10^{6} \mathrm{~N}$ & $K_{t}$ & $1.3 \times 10^{6} \mathrm{jKm}^{-1} \mathrm{~s}^{-1}$ \\
\hline$t_{0}$ & 0.03 & $t_{1}$ & 0.04 \\
\hline$\lambda_{1}^{*}$ & $500 \times 10^{6} \mathrm{Nm}^{-2}$ & $\mu_{1}^{\prime *}$ & $300 \times 10^{6} \mathrm{Nm}^{-2}$ \\
\hline$\alpha_{1}^{* *}$ & $2.0 \times 10^{6} \mathrm{~N}$ & $\beta_{1}^{\prime *}$ & $4.0 \times 10^{6} \mathrm{~N}$ \\
\hline$\gamma_{1}^{* *}$ & $5.0 \times 10^{6} \mathrm{~N}$ & $K_{1}^{\prime *}$ & $0.0149 \times 10^{6} \mathrm{~N}$ \\
\hline$T_{0}$ & $1.293 \times 10^{3} \mathrm{~K}$ & $c$ & $2.04 \times 10^{3} \mathrm{JKg}^{-1} \mathrm{~K}^{-1}$ \\
\hline
\end{tabular}




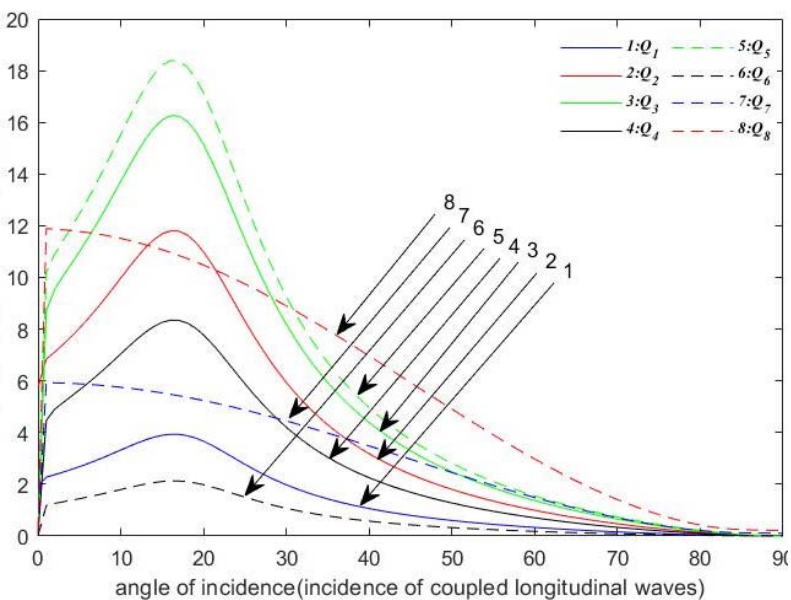

Fig.2. The magnitude of reflection coefficients versus the angle of incidence (incidence of coupled longitudinal waves) under the LStheory.

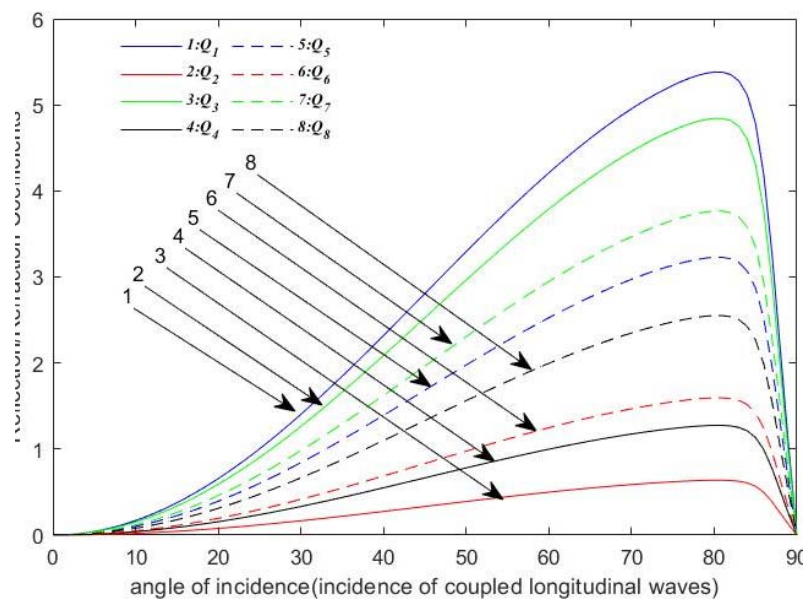

Fig.4. The magnitude of reflection coefficients versus the angle of incidence (incidence of coupled longitudinal waves) under the GLtheory.

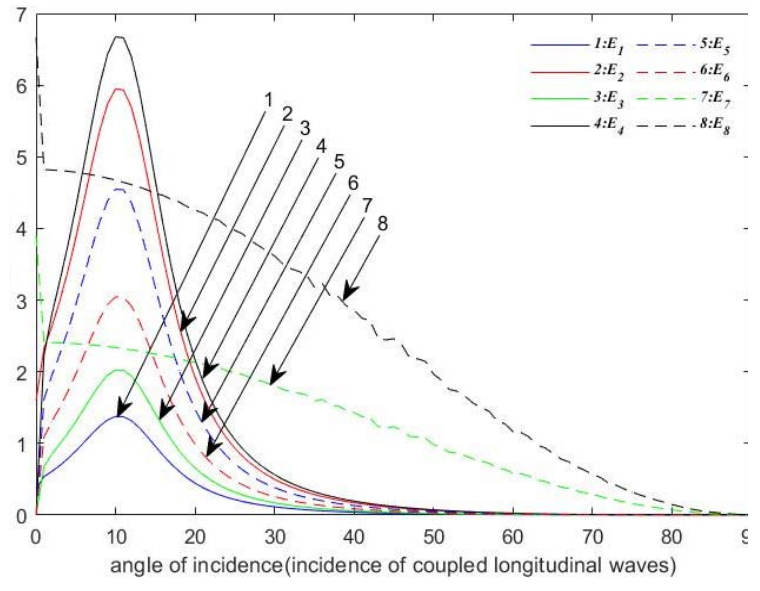

Fig.3. The magnitude of energy coefficients versus the angle of incidence (incidence of coupled longitudinal waves) under the LS-theory.

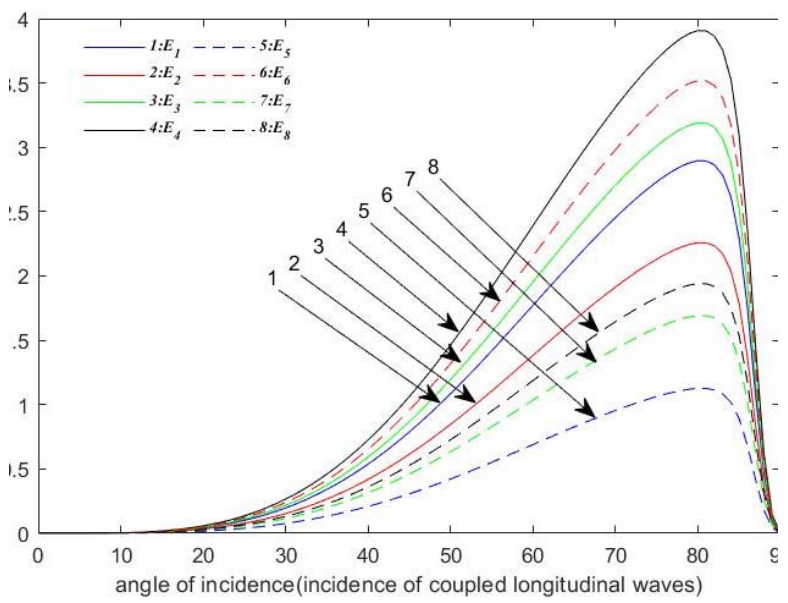

Fig.5. The magnitude of energy coefficients versus the angle of incidence (incidence of coupled longitudinal waves) under the GL-theory.

Figure 2 illustrates that the $Q_{s}(s=1,2 \ldots 6)$ increase sharply for $0^{\circ} \leq \theta_{0} \leq 10$ and after obtaining maxima at $\theta_{0}=10^{\circ}$, decrease gradually as $\theta_{0}$ increases for $10^{\circ} \leq \theta_{0} \leq 90^{\circ} . Q_{7}$ and $Q_{8}$ decrease continuously as $\theta_{0}$ increases for $0^{\circ} \leq \theta_{0} \leq 90^{\circ}$ and attain their maximum value at grazing incidence and vanish at grazing incidence. Figures 2 and 3 illustrate that the graphical behaviour of the reflection and refraction coefficients $\left(Q_{S}\right)$ is similar to their corresponding energy coefficients $\left(E_{S}\right)$.

Figure 4 illustrates that the absolute value of reflection and refraction coefficients $\left(Q_{s}\right)(s=1,2 \ldots 8)$ increase continuously and after that attain their maximum value at $\theta_{0}=85^{\circ}$, it decreases sharply for $85^{\circ} \leq \theta_{0} \leq 90^{\circ}$. Figures 4 and 5 illustrate that the graphical behaviour of the reflection and refraction coefficients $\left(Q_{s}\right)$ is 
similar to their corresponding energy coefficients $\left(E_{s}\right)$. The reflection and refraction coefficients with their corresponding energy ratios are vanishing at grazing as well as normal incidence under GL-theory.

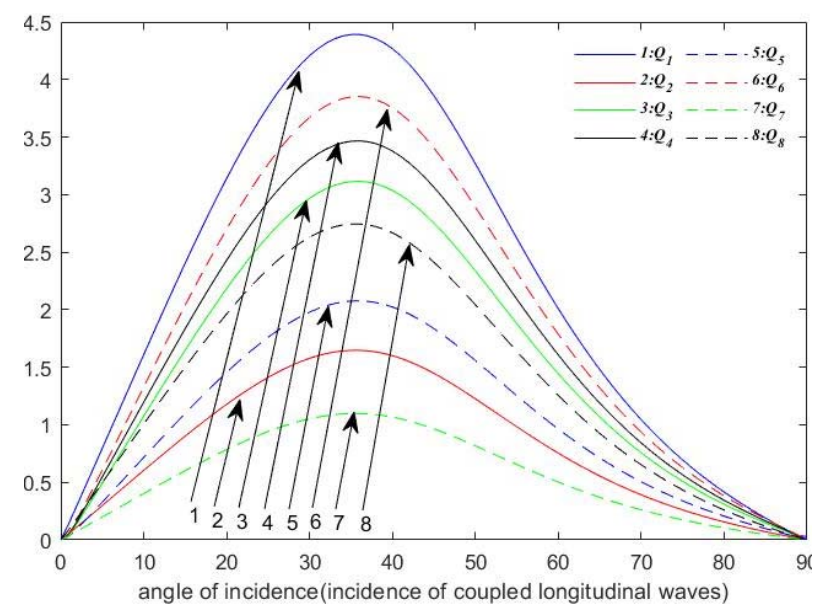

Fig.6. The magnitude of reflection coefficients versus the angle of incidence (incidence of coupled longitudinal waves) under the CTtheory.

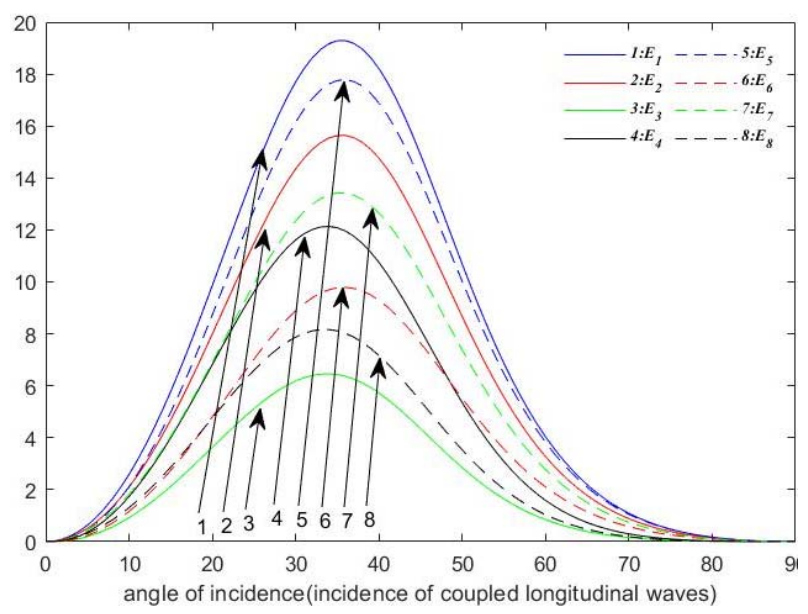

Fig.7. The magnitude of energy coefficients versus the angle of incidence (incidence of coupled longitudinal waves) under the CT-theory.

Figure 6 illustrates that that the absolute value of reflection and refraction coefficients $\left(Q_{s}\right)(s=1,2 \ldots 8)$ increase continuously for $0^{\circ} \leq \theta_{0} \leq 60^{\circ}$ and after attaining maxima at $\theta_{0}=60^{\circ}$, the curves go down and further increase as $\theta_{0}$ increases for $60^{\circ} \leq \theta_{0} \leq 90^{\circ}$. Figures 6 and 7 illustrate that the graphical behaviour of the reflection and refraction coefficients $\left(Q_{S}\right)$ is similar to their corresponding energy coefficients $\left(E_{S}\right)$. The reflection and refraction coefficients with their corresponding energy ratios are vanishing at grazing as well as normal incidence under CT-theory.

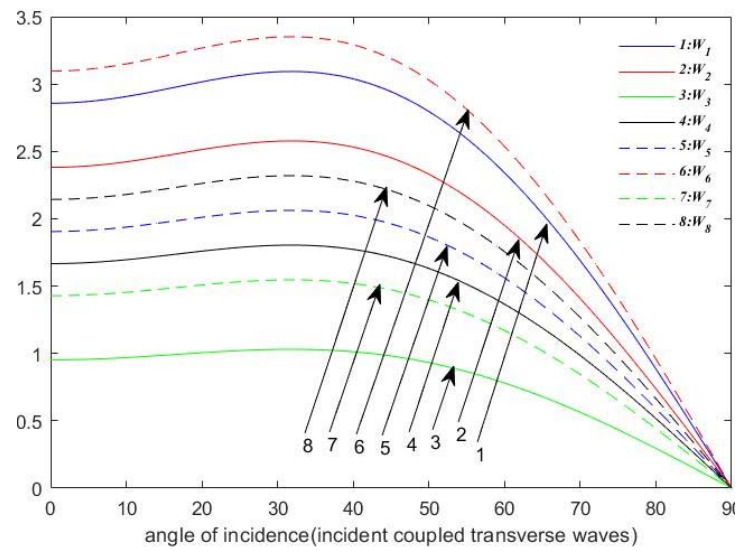

Fig.8. The magnitude of reflection coefficients versus the angle of incidence (incidence of coupled transverse wave) under the LStheory.

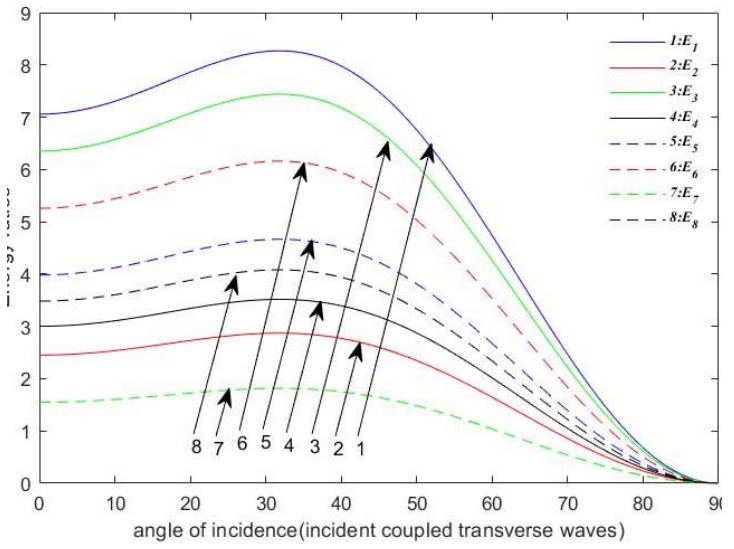

Fig.9. The magnitude of energy coefficients versus the angle of incidence (incidence of coupled transverse wave) under the LS-theory. 
Figures 8 and 9 illustrate that the absolute value of $W_{s}$ with their corresponding energy ratios $E_{s}(s=1,2 \ldots 8)$ decrease continuously as $\theta_{0}$ increases for $0^{\circ} \leq \theta_{0} \leq 90^{\circ}$ and attaining their maximum values at normal incidence. All the reflection and refraction coefficients with their corresponding energy coefficients are disappear at normal incidence under LS-theory.

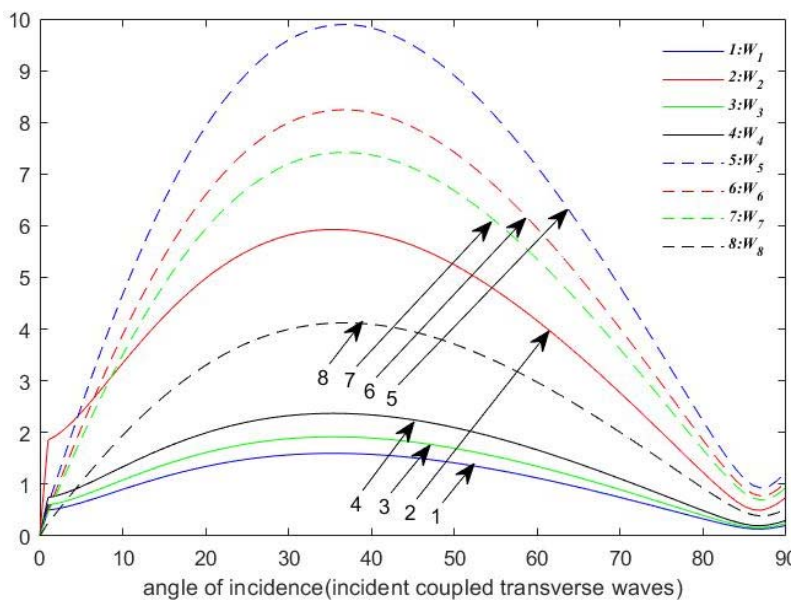

Fig.10. The magnitude of reflection coefficients versus the angle of incidence (incidence of coupled transverse wave) under the GLtheory.

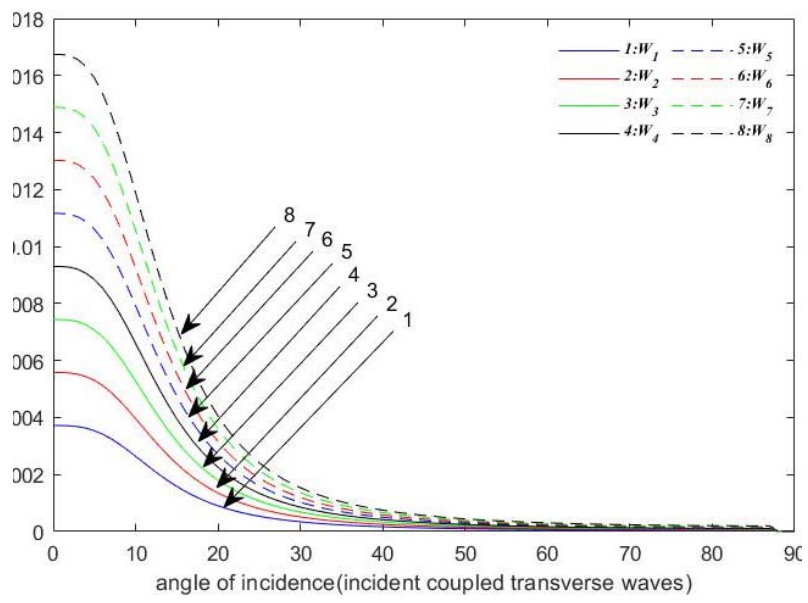

Fig.12. The magnitude of reflection coefficients versus the angle of incidence (incidence of coupled transverse wave) under the CTtheory.

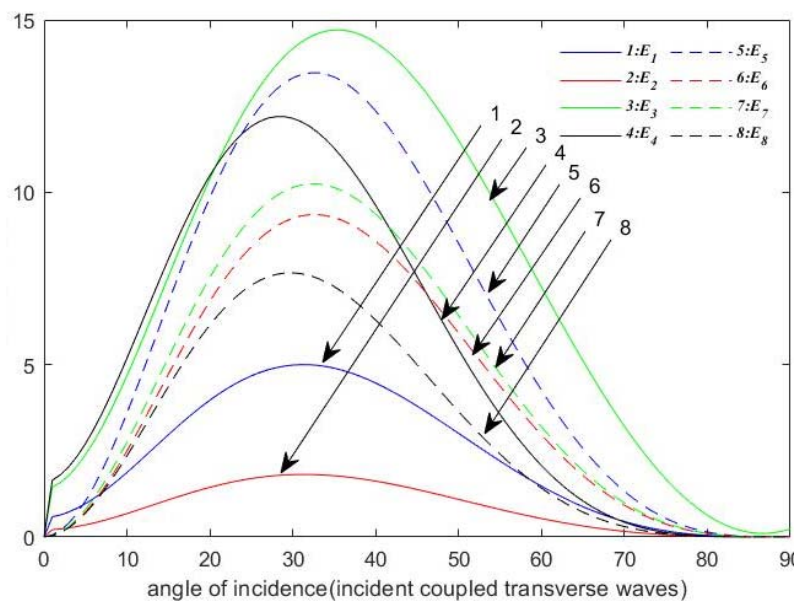

Fig.11. The magnitude of energy coefficients versus the angle of incidence (incidence of coupled transverse wave) under the GL-theory.

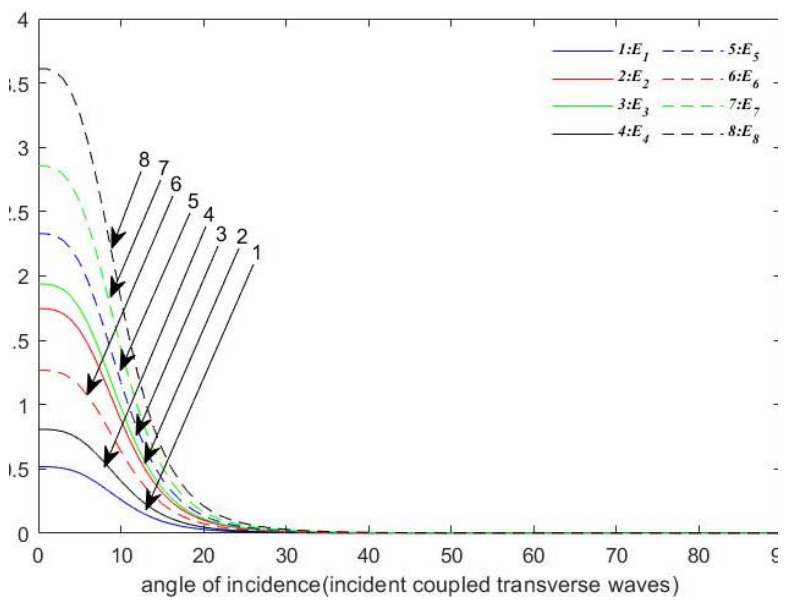

Fig.13. The magnitude of energy coefficients versus the angle of incidence (incidence of coupled transverse wave) under the CT-theory.

Figures 10-11 illustrates that the magnitude of $W_{s}$ with their corresponding energy ratios $E_{s}(s=1,2 \ldots 8)$ against angle of incidence $\theta_{0}$ moving continuously and after attaining their maximum value in the middle of angle of incidence, it decreases gradually. All the reflection and refraction coefficients with their corresponding energy coefficients are disappear at grazing as well as normal incidence under GL-theory. 
Figures 12-13 illustrates that the magnitude of $W_{s}$ with their corresponding energy ratios $E_{s}(s=1,2 \ldots 8)$ are decreases continuously with an increasing value of $\theta_{0}$ for $0^{\circ} \leq \theta_{0} \leq 90^{\circ}$. All the reflection and refraction coefficients with their corresponding energy coefficients disappear at normal incidence and attain their maximum value at grazing incidence under CT-theory.

\section{Conclusions}

We have examined the effect of three theories of thermoelasticity, namely, Green-Lindsay theory (GL), Lord- Shulman theory (LS) and Coupled theory (CT) on the propagation of set of two coupled transverse waves and set of two coupled longitudinal waves in a couple stress micropolar thermoviscous elastic solid medium. We have also examined the reflection and refraction phenomenon of wave propagation in which the plane interface divides a given medium into two half spaces. Here, two relaxation times have been set forth via Kronecker's delta in the equations and constitutive relations for the three theories of thermoelasticity. After reflection and refraction, we find that there exist five waves, namely, longitudinal microrotational wave, set of two coupled transverse waves and set of two coupled longitudinal waves that transmit with various speeds.

The major consequences are obtained as follows

1. The velocity, reflection-refraction coefficients with their corresponding energy ratios of coupled waves are calculated.

2. When coupled longitudinal waves are incident, the coefficients of all the coupled longitudinal and transverse waves are vanishing at normal as well as grazing incidence under GL- and CT- theory. But under LS-theory, the refraction coefficients of coupled transverse waves with their energy ratios are vanishing at normal incidence only.

3. When coupled transverse waves are incident, the reflection and refraction coefficients with their corresponding energy ratios are vanishing at normal incidence only and attain their maximum value at grazing incidence under LS- and CT- theory. But under GL- theory, all the coefficients are vanishing at normal as well as grazing incidence and attain their maximum value in the middle of angle of incidence.

\section{Acknowledgement}

One of the authors $(1, b)$ is grateful to Deenbandhu Chhotu ram University of Science and Technology for awarding a scholarship in the form of URS. The authors are grateful to reviewers for their suggestions for the improvement of the paper.

\section{NOMENCLATURE}

$$
\begin{aligned}
a K_{t}, K^{\prime} & - \text { material constants } \\
e_{k l}=u_{l . k}+e_{l k m} \dot{\phi}_{, i} & - \text { relative tensor } \\
m_{i j} & - \text { couple stress tensor } \\
t_{i j} & - \text { stress tensor } \\
T_{0} & - \text { ambient temperature } \\
t_{0}, t_{l} & - \text { relaxation times } \\
\mathrm{u} & - \text { displacement vector } \\
Y_{i} & - \text { heat flux vector } \\
\alpha^{\prime}, \beta^{\prime}, \gamma^{\prime}, \lambda^{* *}, \mu^{* *} & - \text { material constants }
\end{aligned}
$$




$$
\begin{aligned}
\alpha^{* *}, \beta^{\prime *}, \gamma^{\prime *}, \vartheta & - \text { material constants } \\
\gamma_{k l}=\phi_{k, l} & - \text { wryness tensor } \\
\lambda^{\prime}, \mu^{\prime} & - \text { Lame's constants } \\
\delta_{i j} & - \text { Kronecker's delta } \\
\phi & - \text { microrotation vector } \\
\nabla^{2} & \text { - Laplacian operator } \\
\eta & - \text { entropy per unit mass } \\
\theta & - \text { change in temperature } \\
\rho_{0} & - \text { density of the medium }
\end{aligned}
$$

\section{Appendix $A_{l}$}

$$
\begin{aligned}
& X_{1}=2 \omega K_{2}-2 K_{2}, \quad X_{2}=2 \zeta_{1} \zeta_{2}-\nabla_{1} \omega^{3}-21 K_{2} \omega \zeta^{*}-2 \omega^{2} \zeta_{2}^{*}-2 \omega^{2} K_{2}^{*}-\omega^{2} \zeta_{2}+K_{2} \zeta_{2}, \\
& X_{3}=\nabla_{1} \omega^{3}-\nabla_{2} \omega^{3}+\imath \nabla_{1}^{*} \omega^{4} \quad Y_{1}=1-\Omega, \quad Y_{2}=(1-\Omega)\left(\zeta_{3}-1 \omega \zeta_{3}^{*}\right)+\nabla_{3} \nabla_{4}, \\
& Y_{3}=k_{1}\left(\zeta_{3}-1 \omega \zeta_{3}^{*}\right), \quad \zeta_{1}=\mu_{2}+K_{2} \quad \zeta_{1}^{*}=\mu_{2}^{*}+K_{2}^{*}, \quad \nabla_{1}=\gamma_{2} \zeta_{1} \quad \nabla_{1}^{*}=\gamma_{2}^{*} \zeta_{1}^{*}, \nabla_{2}=\gamma_{2} \zeta_{1}^{*}, \\
& \nabla_{2}^{*}=\gamma_{2}^{*} \zeta_{1}, \quad \zeta_{2}=K_{2}+1 \omega K_{2}^{*}, \quad \zeta_{2}^{*}=\gamma_{2}+1 \omega \gamma_{2}^{*}, \quad \Omega=1-1 n_{1} \delta_{1 k} \omega, \quad \zeta_{3}=\lambda_{2}+\mu_{2}+K_{2}, \\
& \zeta_{3}^{*}=\lambda_{2}^{*}+\mu_{2}^{*}+K_{2}^{*}, \quad \nabla_{3}=1\left(\frac{\vartheta_{1}^{2}}{\tau_{2}}+\frac{\vartheta_{1}^{2}}{\tau_{3}}\right) \delta_{1 k} \omega^{4}, \quad \nabla_{4}=\vartheta_{1}+\vartheta_{2} \delta_{2 k} \omega^{3}, C=\left(\alpha_{2}+\beta_{2}+\gamma_{2}\right)-1 \omega\left(\alpha_{2}^{*}+\beta_{2}^{*}+\gamma_{2}^{*}\right) \\
& \zeta_{2}^{\prime *}=\gamma_{2}^{\prime}+\omega \gamma_{2}^{* *}, \zeta_{4}=\lambda_{2}+1 \omega \lambda_{2}^{*}, \quad \zeta_{5}=2\left(\mu_{2}+1 \omega \mu_{2}^{*}\right)+K_{2}+1 \omega K_{2}^{*}, \zeta_{4}^{\prime}=\lambda_{2}^{\prime}+1 \omega \lambda_{2}^{\prime *}, \\
& h_{11}=1, \quad h_{12}=\left[\zeta_{4}+\zeta_{5}\left(1-s_{21}^{2} \sin ^{2} \theta_{0}\right)+\frac{\nabla_{4} \varkappa_{2}}{l_{2}^{2}}\right] / D_{1} s_{21}^{2}, \quad h_{1 s}=\zeta_{5} \sin \theta_{0} \sqrt{\left(1-s_{S I}^{2} \sin ^{2} \theta_{0}\right)} / D_{I} s_{s l}, \\
& h_{l t}=-\left[\zeta_{4}^{\prime}+\zeta_{5}^{\prime}\left(1-S_{t 1}^{\prime 2} \sin ^{2} \theta_{0}\right)+\nabla_{4}^{\prime} \varkappa_{t}^{\prime}\right] / D_{I} S_{t 1}^{\prime 2}, \quad h_{I q}=\zeta_{5}^{\prime} \sin \theta_{0} \sqrt{\left(1-S_{q 1}^{\prime 2} \sin ^{2} \theta_{0}\right)} / D_{I} S_{q 1}^{\prime}, \\
& h_{21}=\sin \theta_{0} \cos \theta_{0}, h_{22}=\sin \theta_{0} \sqrt{\left(1-s_{21}^{2} \sin ^{2} \theta_{0}\right)} / s_{21}, \\
& h_{2 s}=-\left[\zeta_{6}\left(1-2 s_{s l}^{2} \sin ^{2} \theta_{0}\right)+\zeta_{7}\left(1-s_{s l}^{2} \sin ^{2} \theta_{0}\right)-\frac{\zeta_{7} \eta_{s}}{l_{s}^{2}}\right] / D_{2} s_{s l}^{2}, \quad h_{2 t}=\zeta_{5}^{\prime} \sin \theta_{0} \sqrt{\left(1-S_{t l}^{\prime 2} \sin ^{2} \theta_{0}\right)} / D_{2} S_{t l}^{\prime}, \\
& h_{2 q}=\left[\zeta_{6}^{\prime}\left(1-2 S_{q 1}^{\prime 2} \sin ^{2} \theta_{0}\right)+\zeta_{7}^{\prime}\left(1-S_{q 1}^{\prime 2} \sin ^{2} \theta_{0}\right)-\zeta_{7}^{\prime} \eta_{q}^{\prime}\right] / D_{2} S_{q 1}^{\prime 2}, \quad h_{33}=\sqrt{1-s_{31}^{2} \sin ^{2} \theta_{0}} / s_{31}, \\
& h_{34}=\eta_{4} \sqrt{1-s_{41}^{2} \sin ^{2} \theta_{0}} / \eta_{3} s_{41}, \quad h_{37}=\zeta_{2}^{* *} \eta_{3}^{\prime} \sqrt{1-s_{31}^{\prime 2} \sin ^{2} \theta_{0}} / s_{31}^{\prime} \zeta_{2}^{*} \eta_{3}, \\
& h_{41}=\cos \theta_{0}, \quad h_{42}=\varkappa_{2} \sqrt{\left(1-s_{21}^{2} \sin ^{2} \theta_{0}\right)} / \varkappa_{1} s_{21} \quad h_{45}=k_{1}^{\prime} \varkappa_{1}^{\prime} \sqrt{1-s_{11}^{\prime 2} \sin ^{2} \theta_{0}} / k_{1} \varkappa_{1} s_{11}^{\prime},
\end{aligned}
$$




$$
\begin{aligned}
& h_{46}=k_{1}^{\prime} \varkappa_{2}^{\prime} \sqrt{1-s_{21}^{\prime 2} \sin ^{2} \theta_{0}} / k_{1} \varkappa_{1} s_{21}^{\prime}, \quad h_{51}=\sin \theta_{0}=h_{52}, h_{5 s}=-\sqrt{\left(1-s_{s 1}^{2} \sin ^{2} \theta_{0}\right)} / s_{s 1}, \\
& h_{55}=-\sin \theta_{0}=h_{56}, \quad h_{5 q}=-\sqrt{\left(1-S_{q 1}^{\prime 2} \sin ^{2} \theta_{0}\right)} / S_{q 1}^{\prime}, \quad h_{61}=\cos \theta_{0}, \quad h_{62}=\sqrt{\left(1-s_{21}^{2} \sin ^{2} \theta_{0}\right)} / s_{21}, \\
& h_{63}=\sin \theta_{0}=h_{64}, \quad h_{6 t}=\sqrt{\left(1-S_{t 1}^{\prime 2} \sin ^{2} \theta_{0}\right)} / S_{t 1}^{\prime}, \quad h_{67}=-\sin \theta_{0}=h_{68}, \quad h_{73}=1, h_{74}=\eta_{4} / \eta_{3}, \\
& h_{77}=-\eta_{3}^{\prime} / \eta_{3}, \quad h_{78}=-\eta_{4}^{\prime} / \eta_{3}, \quad h_{81}=1, \quad h_{82}=\varkappa_{2} / \varkappa_{1}, \quad h_{85}=-\varkappa_{1}^{\prime} / \varkappa_{1}, \quad h_{86}=-\varkappa_{2}^{\prime} / \varkappa_{1}, \\
& s=3,4, \quad t=5,6, \quad q=7,8, \quad s_{p l}=\frac{S_{p}}{S_{1}} \quad(p=2,3,4), \quad s_{r l}^{\prime}=\frac{S_{r}^{\prime}}{S_{1}} \quad(r=1,2,3,4), \\
& S_{51}^{\prime}=s_{11}^{\prime}, \quad S_{61}^{\prime}=s_{21}^{\prime}, \quad S_{71}^{\prime}=s_{31}^{\prime}, \quad S_{81}^{\prime}=s_{41}^{\prime}, \quad \varkappa_{5}^{\prime}=\frac{\varkappa_{1}^{\prime}}{l_{1}^{\prime 2}}, \quad \varkappa_{6}^{\prime}=\frac{\varkappa_{2}^{\prime}}{l_{2}^{\prime 2}}, \quad \eta_{7}^{\prime}=\frac{\eta_{3}^{\prime}}{l_{3}^{\prime 2}}, \quad \eta_{8}^{\prime}=\frac{\eta_{4}^{\prime}}{l_{4}^{\prime 2}}, \\
& \nabla_{4}^{\prime}=\vartheta_{1}^{\prime}+\vartheta_{2}^{\prime} \delta_{2 k} \omega^{3}, \quad D_{1}=\left[\zeta_{4}+\zeta_{5} \cos ^{2} \theta_{0}+\frac{\nabla_{4} \varkappa_{1}}{l_{1}^{2}}\right], \quad D_{2}=\zeta_{5}, \quad Z_{1}=-1, \quad Z_{2}=\sin \theta_{0} \cos \theta_{0} \\
& Z_{3}=0, \quad Z_{4}=\cos \theta_{0}, \quad Z_{5}=-\sin \theta_{0}, \quad Z_{6}=\cos \theta_{0}, \quad Z_{7}=0, \quad Z_{8}=-1, \quad \zeta_{6}=\mu_{2}+1 \omega \mu_{2}^{*} \\
& \zeta_{5}^{\prime}=2\left(\mu_{2}^{\prime}+\imath \omega^{\prime \prime \mu_{2}^{*}}\right)+K_{2}^{\prime}+\imath \omega^{\prime} K_{2}^{\prime *}, \quad \zeta_{6}^{\prime}=\mu_{2}^{\prime}+\imath \omega \mu_{2}^{\prime *}, \quad \zeta_{7}=K_{2}+\imath \omega K_{2}^{*}, \quad \zeta_{7}^{\prime}=K_{2}^{\prime}+\imath \omega K_{2}^{\prime *}, \\
& E_{1}=-Q_{1}^{2}, \quad E_{2}=Q_{2}^{2} L_{1}\left[\zeta_{4}+\zeta_{5}+\frac{\nabla_{4} \varkappa_{2}}{l_{2}^{2}}-\frac{\zeta_{7} \varkappa_{2}^{2}}{l_{2}^{2}}\right] l_{2}^{3} \cos \theta, \quad h_{38}=\zeta_{2}^{* *} \eta_{4}^{\prime} \sqrt{1-s_{41}^{\prime 2} \sin ^{2} \theta_{0}} / s_{41}^{\prime} \zeta_{2}^{*} \eta_{3} \\
& E_{3,4}=Q_{3,4}^{2} L_{1}\left[\zeta_{6}+\zeta_{7}-\frac{\eta_{3,4}}{l_{3,4}^{2}}\left(\zeta_{2}^{*} \eta_{3,4}+\zeta_{7}\right)\right] l_{3,4}^{3} \cos \theta_{3,4} \\
& E_{5,6}=Q_{5,6}^{2} L_{1}\left[\zeta_{4}^{\prime}+\zeta_{5}^{\prime}+\frac{\nabla_{4}^{\prime} \varkappa_{1,2}^{\prime}}{l_{1,2}^{\prime 2}}-\frac{k_{1}^{\prime} \varkappa_{1,2}^{\prime 2}}{l_{l, 2}^{\prime 2}}\right] l_{l, 2}^{\prime 3} \cos \theta_{1,2}^{\prime} . \\
& E_{7,8}=Q_{7,8}^{2} L_{1}\left[\zeta_{6}^{\prime}+\zeta_{7}^{\prime}-\frac{\eta_{3,4}^{\prime}}{l_{3,4}^{\prime 2}}\left(\zeta_{2}^{*} \eta_{3,4}^{\prime}+\zeta_{7}^{\prime}\right)\right] l_{3,4}^{3} \cos \theta_{3,4}^{\prime}, \quad L_{1}=\left[\left(\zeta_{4}+\zeta_{5}+\frac{\nabla_{4} \varkappa_{1}}{l_{2}^{2}}-\frac{\zeta_{7} \varkappa_{1}^{2}}{l_{2}^{2}}\right) l_{1}^{3} \cos \theta_{1}\right]^{-1} .
\end{aligned}
$$

\section{Appendix $\boldsymbol{A}_{2}$}

$$
\begin{aligned}
& h_{11}=\left[\zeta_{4}+\zeta_{5}\left(1-s_{12}^{2} \sin ^{2} \theta_{0}\right)+\frac{\nabla_{4} \varkappa_{1}}{l_{2}^{2}}\right] / D_{3} s_{12}^{2}, \quad h_{12}=1, \quad h_{1 s}=\zeta_{5} \sin \theta_{0} \sqrt{\left(1-s_{s 2}^{2} \sin ^{2} \theta_{0}\right)} / D_{3} s_{s 2}, \\
& h_{1 t}=-\left[\zeta_{4}^{\prime}+\zeta_{5}^{\prime}\left(1-S_{t 2}^{\prime 2} \sin ^{2} \theta_{0}\right)+\nabla_{4} \varkappa_{t}^{\prime}\right] / D_{3} S_{t 2}^{\prime 2}, \quad h_{1 q}=\zeta_{5}^{\prime} \sin \theta_{0} \sqrt{\left(1-S_{q 2}^{\prime 2} \sin ^{2} \theta_{0}\right)} / D_{1} S_{q 2}^{\prime} \\
& h_{21}=\sin \theta_{0} \sqrt{\left(1-s_{12}^{2} \sin ^{2} \theta_{0}\right) \backslash B i g / s_{12},} \quad h_{22}=\sin \theta_{0} \cos \theta_{0}
\end{aligned}
$$




$$
\begin{aligned}
& h_{2 s}=-\left[\zeta_{6}\left(1-2 s_{s 2}^{2} \sin ^{2} \theta_{0}\right)+\zeta_{7}\left(1-s_{s 2}^{2} \sin ^{2} \theta_{0}\right)-\frac{\zeta_{7} \eta_{s}}{l_{s}^{2}}\right] / D_{2} s_{s 2}^{2} \\
& h_{2 t}=\zeta_{5}^{\prime} \sin \theta_{0} \sqrt{\left(1-S_{t 2}^{\prime 2} \sin ^{2} \theta_{0}\right)} / D_{2} S_{t 2}^{\prime}, \quad h_{33}=\sqrt{1-s_{32}^{2} \sin ^{2} \theta_{0}} / s_{32}, \\
& h_{2 q}=\left[\zeta_{6}^{\prime}\left(1-2 S_{q 2}^{\prime 2} \sin ^{2} \theta_{0}\right)+\zeta_{7}^{\prime}\left(1-S_{q 2}^{\prime 2} \sin ^{2} \theta_{0}\right)-\zeta_{7}^{\prime} \eta_{q}^{\prime}\right] / D_{2} S_{q 2}^{\prime 2}, \\
& h_{34}=\eta_{4} \sqrt{1-s_{42}^{2} \sin ^{2} \theta_{0}} / \eta_{3} s_{42}, \quad h_{37}=\zeta_{2}^{* *} \eta_{3}^{\prime} \sqrt{1-s_{32}^{\prime 2} \sin ^{2} \theta_{0}} / s_{32}^{\prime} \zeta_{2}^{*} \eta_{3}, \\
& h_{41}=\varkappa_{1} \sqrt{\left(1-s_{12}^{2} \sin ^{2} \theta_{0}\right)} / \zeta_{2} s_{12}, \quad h_{42}=\cos \theta_{0}, \quad h_{45}=k_{1}^{\prime} \varkappa_{1}^{\prime} \sqrt{1-s_{12}^{\prime 2} \sin ^{2} \theta_{0}} / k_{1} \varkappa_{1} s_{12}^{\prime}, \\
& h_{46}=k_{1}^{\prime} \varkappa_{2}^{\prime} \sqrt{1-s_{22}^{\prime 2} \sin ^{2} \theta_{0}} / k_{1} \varkappa_{1} s_{21}^{\prime}, \quad h_{51}=\sin \theta_{0}=h_{52}, \quad h_{5 s}=-\sqrt{\left(1-s_{s 2}^{2} \sin ^{2} \theta_{0}\right)} / s_{s 2}, \\
& h_{55}=-\sin \theta_{0}=h_{56}, \quad h_{5 q}=-\sqrt{\left(1-S_{q 2}^{\prime 2} \sin ^{2} \theta_{0}\right)} / S_{q 2}^{\prime}, \quad h_{61}=\sqrt{\left(1-s_{12}^{2} \sin ^{2} \theta_{0}\right)} / s_{12}, \quad h_{62}=\cos \theta_{0} \\
& h_{63}=\sin \theta_{0}=h_{64}, \quad h_{6 t}=\sqrt{\left(1-S_{t 2}^{\prime 2} \sin ^{2} \theta_{0}\right)} / S_{t 2}^{\prime}, \quad h_{67}=-\sin \theta_{0}=h_{68}, \quad h_{73}=1, \quad h_{74}=\eta_{4} / \eta_{3}, \\
& h_{77}=-\eta_{3}^{\prime} / \eta_{3}, \quad h_{78}=-\eta_{4}^{\prime} / \eta_{3}, \quad h_{81}=\varkappa_{1} / \varkappa_{2}, \quad h_{82}=1, \quad h_{38}=\zeta_{2}^{* *} \eta_{4}^{\prime} \sqrt{1-s_{42}^{\prime 2} \sin ^{2} \theta_{0}} / s_{42}^{\prime} \zeta_{2}^{*} \eta_{3}, \\
& h_{85}=-\varkappa_{1}^{\prime} / \varkappa_{1}, \quad h_{86}=-\varkappa_{2}^{\prime} / \varkappa_{1} \quad s_{p 2}=\frac{S_{p}}{S_{2}} \quad(p=1,3,4), \quad s_{r 2}^{\prime}=\frac{S_{r}^{\prime}}{S_{2}} \quad(r=1,2,3,4), \\
& S_{52}^{\prime}=s_{12}^{\prime}, \quad S_{62}^{\prime}=s_{22}^{\prime}, \quad S_{72}^{\prime}=s_{32}^{\prime}, \quad S_{82}^{\prime}=s_{42}^{\prime}, \quad D_{3}=\left[\zeta_{4}+\zeta_{5} \cos ^{2} \theta_{0}+\frac{\nabla_{4} \varkappa_{2}}{l_{2}^{2}}\right], \\
& E_{1}=Q_{1}^{2} \mathrm{~L}_{2}\left[\zeta_{4}+\zeta_{5}+\frac{\nabla_{4} \varkappa_{1}}{l_{1}^{2}}-\frac{\zeta_{7} \varkappa_{1}^{2}}{l_{1}^{2}}\right] l_{1}^{3} \cos \theta_{1}, \quad E_{3,4}=Q_{3,4}^{2} \mathrm{~L}_{2}\left[\zeta_{6}+\zeta_{7}-\frac{\eta_{3,4}}{l_{3,4}^{2}}\left(\zeta_{2}^{*} \eta_{3,4}+\zeta_{7}\right)\right] l_{3,4}^{3} \cos \theta_{3,4} \\
& E_{2}=Q_{2}^{2}, \quad E_{5,6}=Q_{5,6}^{2} \mathrm{~L}_{2}\left[\zeta_{4}^{\prime}+\zeta_{5}^{\prime}+\frac{\nabla_{4}^{\prime} \varkappa_{1,2}^{\prime}}{l_{1,2}^{\prime 2}}-\frac{k_{1}^{\prime} \varkappa_{1,2}^{\prime 2}}{l_{l, 2}^{\prime 2}}\right] l_{1,2}^{\prime 3} \cos \theta_{1,2}^{\prime}, \\
& E_{7,8}=Q_{7,8}^{2} L_{2}\left[\zeta_{6}^{\prime}+\zeta_{7}^{\prime}-\frac{\eta_{3,4}^{\prime}}{l_{3,4}^{\prime 2}}\left(\zeta_{2}^{*} \eta_{3,4}^{\prime}+\zeta_{7}^{\prime}\right)\right] l_{3,4}^{\prime 3} \cos \theta_{3,4}^{\prime}, \quad L_{2}=\left[\left(\zeta_{4}+\zeta_{5}+\frac{\nabla_{4} \varkappa_{2}}{l_{2}^{2}}-\frac{\zeta_{7} \varkappa_{2}^{2}}{l_{2}^{2}}\right) l_{2}^{3} \cos \theta_{2}\right]^{-1} .
\end{aligned}
$$

\section{Appendix $B_{1}$}

$$
\begin{aligned}
& U_{1 s}=\left[\zeta_{4}+\zeta_{5}\left(1-s_{s 3}^{2} \sin ^{2} \theta_{0}\right)+\frac{\nabla_{4} \varkappa_{s}}{l_{s}^{2}}\right] / D_{2} s_{s 3}^{2}, \quad U_{13}=\sin \theta_{0} \cos \theta_{0}, \quad U_{14}=\sin \theta_{0} \sqrt{\left(1-s_{43}^{2} \sin ^{2} \theta_{0}\right)} / s_{43}, \\
& U_{1 t}=-\left[\zeta_{4}^{\prime}+\zeta_{5}^{\prime}\left(1-S_{t 3}^{\prime 2} \sin ^{2} \theta_{0}\right)+\nabla_{4}^{\prime} \varkappa_{t}^{\prime}\right] / D_{2} S_{t 3}^{\prime 2}, \quad U_{1 q}=\zeta_{5}^{\prime} \sin \theta_{0} \sqrt{\left(1-S_{q 3}^{\prime 2} \sin ^{2} \theta_{0}\right)} / D_{2} S_{q 3}^{\prime},
\end{aligned}
$$




$$
\begin{aligned}
& U_{2 s}=\zeta_{5} \sin \theta_{0} \sqrt{\left(1-s_{s 3}^{2} \sin ^{2} \theta_{0}\right)} / D_{4} s_{s 3}, \quad U_{23}=-1, \quad U_{2 t}=\zeta_{6}^{\prime} \sin \theta_{0} \sqrt{\left(1-S_{t 3}^{\prime 2} \sin ^{2} \theta_{0}\right)} / D_{4} S_{t 3}^{\prime}, \\
& U_{24}=-\left[\zeta_{6}\left(1-2 s_{43}^{2} \sin ^{2} \theta_{0}\right)+\zeta_{7}\left(1-s_{43}^{2} \sin ^{2} \theta_{0}\right)-\frac{\zeta_{7} \eta_{4}}{l_{4}^{2}}\right] / D_{4} s_{43}^{2}, \quad U_{33}=\cos \theta_{0}, \\
& U_{2 q}=\left[\zeta_{6}^{\prime}\left(1-2 S_{q 3}^{\prime 2} \sin ^{2} \theta_{0}\right)+\zeta_{7}^{\prime}\left(1-S_{q 3}^{\prime 2} \sin ^{2} \theta_{0}\right)-\zeta_{7}^{\prime} \eta_{q}^{\prime}\right] / D_{4} S_{q 3}^{\prime 2}, \quad U_{34}=\eta_{4} \sqrt{1-s_{43}^{2} \sin ^{2} \theta_{0}} / \eta_{3} s_{43}, \\
& U_{37}=\zeta_{2}^{* *} \eta_{3}^{\prime} \sqrt{1-s_{33}^{\prime 2} \sin ^{2} \theta_{0}} / s_{33}^{\prime} \zeta_{2}^{*} \eta_{3}, \quad U_{38}=\zeta_{2}^{*} \eta_{4}^{\prime} \sqrt{1-s_{43}^{\prime 2} \sin ^{2} \theta_{0}} / s_{43}^{\prime} \zeta_{2}^{*} \eta_{3}, \\
& U_{51}=\sin \theta_{0}=U_{52}, \quad U_{4 s}=\sqrt{\left(1-s_{s 3}^{2} \sin ^{2} \theta_{0}\right)} / s_{s 3}, \quad U_{45}=\zeta_{2}^{*} \varkappa_{1}^{\prime} \sqrt{1-s_{13}^{\prime 2} \sin ^{2} \theta_{0}} / \zeta^{*} \varkappa_{1} s_{13}^{\prime}, \\
& U_{53}=-\cos \theta_{0}, \quad U_{46}=k_{1}^{\prime} \zeta_{2}^{\prime} \sqrt{1-s_{23}^{\prime 2} \sin ^{2} \theta_{0}} / k_{1} \zeta_{1} s_{23}^{\prime}, \quad U_{54}=-\sqrt{\left(1-s_{43}^{2} \sin ^{2} \theta_{0}\right)} / s_{43}, \\
& U_{55}=-\sin \theta_{0}=U_{56}, \quad U_{5 q}=-\sqrt{\left(1-S_{q 3}^{\prime 2} \sin ^{2} \theta_{0}\right)} / S_{q 3}^{\prime}, \quad U_{6 s}=-\sqrt{\left(1-s_{s 3}^{2} \sin ^{2} \theta_{0}\right)} / s_{s 3}, \\
& h_{63}=-\sin \theta_{0}=U_{64}, \quad U_{6 t}=-\sqrt{\left(1-S_{t 3}^{\prime 2} \sin ^{2} \theta_{0}\right)} / S_{t 3}^{\prime}, \quad U_{67}=\sin \theta_{0}=U_{68}, \\
& U_{73}=1, \quad U_{74}=\eta_{4} / \eta_{3}, \quad U_{77}=-\eta_{3}^{\prime} / \eta_{3}, \quad U_{78}=-\eta_{4}^{\prime} / \eta_{3}, \quad U_{81}=1, \quad U_{82}=\varkappa_{2} / \varkappa_{1}, \\
& U_{85}=-\varkappa_{1}^{\prime} / \varkappa_{1}, \quad U_{86}=-\varkappa_{2}^{\prime} / \varkappa_{1}, \quad W_{1}=\sin \theta_{0} \cos \theta_{0}, \quad W_{2}=1, \quad W_{3}=\cos \theta_{0}, \\
& W_{4}=0, \quad W_{5}=-\cos \theta_{0}, \quad W_{6}=\sin \theta_{0}, \quad W_{7}=-1, \quad W_{8}=0 .
\end{aligned}
$$

Here now

$$
\begin{aligned}
& s=1,2 ; \quad s_{p 3}=\frac{S_{p}}{S_{3}} \quad(p=1,2,4), \quad s_{r 3}^{\prime}=\frac{S_{r}^{\prime}}{S_{3}} \quad(r=1,2,3,4), \quad S_{53}^{\prime}=s_{13}^{\prime}, \quad S_{63}^{\prime}=s_{23}^{\prime}, \\
& S_{73}^{\prime}=s_{33}^{\prime}, \quad S_{83}^{\prime}=s_{43}^{\prime}, \quad D_{4}=-\left[\zeta_{6} \cos 2 \theta_{0}+\zeta_{7} \cos ^{2} \theta_{0}-\frac{\zeta_{7} \eta_{3}}{l_{3}^{2}}\right], \\
& E_{1,2}=W_{1,2}^{2} \mathrm{~L}_{3}\left[\zeta_{4}+\zeta_{5}+\frac{\nabla_{4} \varkappa_{1,2}}{l_{1,2}^{2}}-\frac{\zeta_{7} \varkappa_{1,2}^{2}}{l_{1,2}^{2}}\right] l_{l, 2}^{3} \cos \theta_{1,2}, \quad E_{3}=W_{3}^{2}, \\
& E_{4}=W_{4}^{2} \mathrm{~L}_{3}\left[\zeta_{6}+\zeta_{7}-\frac{\eta_{4}}{l_{4}^{2}}\left(\zeta_{2}^{*} \eta_{4}+\zeta_{7}\right)\right] l_{4}^{3} \cos \theta_{4}, \quad E_{5,6}=W_{5,6}^{2} L_{3}\left[\zeta_{4}^{\prime}+\zeta_{5}^{\prime}+\frac{\nabla_{4}^{\prime} \varkappa_{1,2}^{\prime}}{l_{1,2}^{\prime 2}}-\frac{k_{1}^{\prime} \varkappa_{1,2}^{\prime 2}}{l_{1,2}^{\prime 2}}\right] l_{1,2}^{\prime 3} \cos \theta_{1,2}^{\prime}, \\
& E_{7,8}=W_{7,8}^{2} L_{3}\left[\zeta_{6}^{\prime}+\zeta_{7}^{\prime}-\frac{\eta_{3,4}^{\prime}}{l_{3,4}^{\prime 2}}\left(\zeta_{2}^{*} \eta_{3,4}^{\prime}+\zeta_{7}^{\prime}\right)\right] l_{3,4}^{\prime 3} \cos \theta_{3,4}^{\prime}, \quad L_{3}=\left[\left(\zeta_{6}+\zeta_{7}-\frac{\eta_{3}}{l_{3}^{2}}\left(\zeta_{2}^{*} \eta_{3}+\zeta_{7}\right)\right) l_{3}^{3} \cos \theta_{0}\right] .
\end{aligned}
$$




\section{Appendix $\boldsymbol{B}_{2}$}

$$
\begin{aligned}
& U_{I s}=\left[\zeta_{4}+\zeta_{5}\left(1-s_{s 4}^{2} \sin ^{2} \theta_{0}\right)+\frac{\nabla_{4} \varkappa_{s}}{l_{s}^{2}}\right] / D_{2} s_{s 4}^{2}, \quad U_{13}=\sin \theta_{0} \sqrt{\left(1-s_{34}^{2} \sin ^{2} \theta_{0}\right)} / s_{34}, \\
& U_{14}=\sin \theta_{0} \cos \theta_{0}, \quad U_{1 t}=-\left[\zeta_{4}^{\prime}+\zeta_{5}^{\prime}\left(1-S_{t 4}^{\prime 2} \sin ^{2} \theta_{0}\right)+\nabla^{\prime}{ }_{4} x_{t}^{\prime}\right] / D_{2} S_{t 4}^{\prime 2}, \quad U_{63}=-\sin \theta_{0}=U_{64}, \\
& U_{1 q}=\zeta_{5}^{\prime} \sin \theta_{0} \sqrt{\left(1-S_{q 4}^{\prime 2} \sin ^{2} \theta_{0}\right)} / D_{2} s_{q 4}^{\prime}, \quad U_{2 s}=\zeta_{5} \sin \theta_{0} \sqrt{\left(1-s_{s 4}^{2} \sin ^{2} \theta_{0}\right)} / D_{4} s_{s 4}, \\
& U_{23}=-\left[\zeta_{6}\left(1-2 s_{34}^{2} \sin ^{2} \theta_{0}\right)+\zeta_{7}\left(1-s_{34}^{2} \sin ^{2} \theta_{0}\right)-\frac{\zeta_{7} \eta_{3}}{l_{3}^{2}}\right] / D_{3} s_{34}^{2}, \quad U_{4 s}=\sqrt{\left(1-s_{s 3}^{2} \sin ^{2} \theta_{0}\right)} / s_{s 3}, \\
& U_{24}=-1, U_{2 t}=\zeta_{6}^{\prime} \sin \theta_{0} \sqrt{\left(1-S_{t 4}^{\prime 2} \sin ^{2} \theta_{0}\right)} / D_{4} s_{t 4}^{\prime}, \quad U_{33}=\eta_{3} \sqrt{1-s_{34}^{2} \sin ^{2} \theta_{0}} / \eta_{4} s_{44}, \quad U_{34}=\cos \theta_{0} \\
& U_{2 q}=\left[\zeta_{6}^{\prime}\left(1-2 S_{q 4}^{\prime 2} \sin ^{2} \theta_{0}\right)+\zeta_{7}^{\prime}\left(1-S_{q 4}^{\prime 2} \sin ^{2} \theta_{0}\right)-\zeta_{7}^{\prime} \eta_{q}^{\prime}\right] / D_{4} S_{q 4}^{\prime 2}, \quad U_{51}=\sin \theta_{0}=U_{52}, \\
& U_{37}=\zeta_{2}^{\prime *} \eta_{3}^{\prime} \sqrt{1-s_{34}^{\prime 2} \sin ^{2} \theta_{0}} / s_{34}^{\prime} \zeta_{2}^{*} \eta_{4}, \quad U_{38}=\zeta_{2}^{*} \eta_{4}^{\prime} \sqrt{1-s_{44}^{\prime 2} \sin ^{2} \theta_{0}} / s_{44}^{\prime} \zeta_{2}^{*} \eta_{4}, \quad U_{54}=-\cos \theta_{0}, \\
& U_{45}=\zeta_{2}^{*} \varkappa_{1}^{\prime} \sqrt{1-s_{14}^{\prime 2} \sin ^{2} \theta_{0}} / \zeta^{*} \varkappa_{1}^{\prime} s_{14}^{\prime}, \quad U_{46}=k_{1}^{\prime} \zeta_{2}^{\prime} \sqrt{1-s_{24}^{\prime 2} \sin ^{2} \theta_{0}} / k_{1} \zeta_{1} s_{24}^{\prime}, \quad U_{55}=-\sin \theta_{0}=U_{56}, \\
& U_{53}=-\sqrt{\left(1-s_{34}^{2} \sin ^{2} \theta_{0}\right)} / s_{34}, \quad U_{5 q}=-\sqrt{\left(1-S_{q 4}^{\prime 2} \sin ^{2} \theta_{0}\right)} / S_{q 4}^{\prime}, \quad U_{6 s}=-\sqrt{\left(1-s_{s 4}^{2} \sin ^{2} \theta_{0}\right)} / s_{s 4}, \\
& U_{6 t}=-\sqrt{\left(1-S_{t 4}^{\prime 2} \sin ^{2} \theta_{0}\right)} / S_{t 4}^{\prime}, \quad U_{67}=\sin \theta_{0}=U_{68}, \quad U_{73}=\eta_{3} / \eta_{4}, \quad U_{74}=1, \\
& U_{77}=-\eta_{3}^{\prime} / \eta_{4}, \quad U_{78}=-\eta_{4}^{\prime} / \eta_{4}, U_{81}=1, \quad U_{82}=\varkappa_{2} / \varkappa_{1}, \quad U_{85}=-\varkappa_{1}^{\prime} / \varkappa_{1}, \quad U_{86}=-\varkappa_{2}^{\prime} / \varkappa_{1} .
\end{aligned}
$$

Here now $\quad s=1,2 ; \quad s_{p 4}=\frac{S_{p}}{S_{4}} \quad(p=1,2,3)$,

$$
\begin{aligned}
& s_{r 4}^{\prime}=\frac{S_{r}^{\prime}}{S_{4}},(r=1,2,3,4), \quad S_{54}^{\prime}=s_{14}^{\prime}, \quad S_{64}^{\prime}=s_{24}^{\prime}, \quad S_{74}^{\prime}=s_{34}^{\prime}, \quad S_{84}^{\prime}=s_{44}^{\prime}, \\
& D_{3}=-\left[\zeta_{6} \cos 2 \theta_{0}-\zeta_{7} \cos ^{2} \theta_{0}-\frac{\zeta_{7} \eta_{4}}{l_{4}^{2}}\right], \quad E_{1,2}=W_{1,2}^{2} \mathrm{~L}_{4}\left[\zeta_{4}+\zeta_{5}+\frac{\nabla_{4} \varkappa_{1,2}}{l_{1,2}^{2}}-\frac{\zeta_{7} \varkappa_{1,2}^{2}}{l_{1,2}^{2}}\right] l_{1,2}^{3} \cos \theta_{1,2}, \\
& E_{3}=W_{3}^{2} \mathrm{~L}_{4}\left[\zeta_{6}+\zeta_{7}-\frac{\eta_{4}}{l_{4}^{2}}\left(\zeta_{2}^{*} \eta_{4}+\zeta_{7}\right)\right] l_{4}^{3} \cos \theta_{4}, \quad E_{4}=W_{4}^{2}, \quad L_{4}=\left[\left(\zeta_{6}+\zeta_{7}-\frac{\eta_{4}}{l_{4}^{2}}\left(\zeta_{2}^{*} \eta_{4}+\zeta_{7}\right)\right) l_{4}^{3} \cos \theta_{0}\right]^{-1}, \\
& E_{5,6}=W_{5,6}^{2} L_{4}\left[\zeta_{4}^{\prime}+\zeta_{5}^{\prime}+\frac{\nabla_{4}^{\prime} \varkappa_{1,2}^{\prime}}{l_{l, 2}^{\prime 2}}-\frac{k_{1}^{\prime} x_{l, 2}^{\prime 2}}{l_{l, 2}^{\prime 2}}\right] l_{1,2}^{\prime 3} \cos \theta_{l, 2}^{\prime}
\end{aligned}
$$




$$
E_{7,8}=W_{7,8}^{2} L_{4}\left[\zeta_{6}^{\prime}+\zeta_{7}^{\prime}-\frac{\eta_{3,4}^{\prime}}{l_{3,4}^{\prime 2}}\left(\zeta_{2}^{*} \eta_{3,4}^{\prime}+\zeta_{7}^{\prime}\right)\right] i_{3,4}^{3} \cos \theta_{3,4}^{\prime} .
$$

\section{Reference}

[1] Eringen A.C. (1966): Linear theory of micropolar elasticity.- J. of Mech. and Mat. Struct., vol.15, pp.909-923.

[2] Eringen A.C. (1970): Foundations of micropolar thermoelasticity.- Int. J. Eng. Sci., vol.23, Springen-Verlag, Berlin.

[3] Nowacki W. (1986): Theory of asymmetric elasticity.- Oxford, Pergamon.

[4] Dost S. and Taborrok B. (1978): Generalised micropolar thermoelasticity.- Int. J. Eng. Sci., vol.16, pp.173-178.

[5] Green A.E. and Lindsay K.A. (1972): Thermoelasticity.- J. of Elas., vol.2, pp.1-7

[6] Chandersekharaiah D.S. (1986): Heat flux dependent micropolar thermoelasticity.- Int. J. Eng. Sci., vol.24, pp.1389-1395.

[7] Chandrasekhariah D.S. (1998): Hyperbolic thermoelasticity.-Appl. Mech. Rev., vol.51, pp.705-729.

[8] Farka I.s and Szekers A. (1984): Application of modified law of heat conduction and state equation to dynamical problem of thermoelasticity.- Periodica Poly. Mech. Eng., vol.28, pp.163-170.

[9] Szekers A. (1980): Equation system of thermoelasticity using the modified law of thermal conductivity.- Periodica Poly. Mech. Eng., vol.24, pp.253-261.

[10] Biot A.M. (1965): Mechanics of incremental deformations.- John Wiley and Sons, New York, p.516.

[11] Hetnarski R.B. and Ignaczak J. (1999): Generalised thermoelasticity.- J. of Ther. Str., vol.22, pp.451-476.

[12] Lord H. and Shulman Y. (1967): A generalised dynamical theory of thermoelasticity.- J. of Mech. and Phy. of Solids, vol.15, pp.299-309.

[13] Hetnarski R.B. and Ignaczak J. (1996): Solution like wave in a low temperature nonlinear thermoelastic solid.- Int. J. Eng. Sci., vol.4, pp.1767-1787.

[14] Green A.E. and. Naghdi P.M. (1993): Thermoelasticity without energy dissipation.- J. Elast., vol.31, pp.189-208.

[15] Tomar S.K. and Khurana A. (2008): Transmission of longitudinal wave at plane interface between micropolar elastic and chiral solid half-spaces: incident from micropolar half-spaces.- J. Of Sound and Vib., vol.311, pp. 973-990.

[16] Tomar S. K. and Khurana A. (2017): Waves in non-elastic solids with voids.- Int. J. of Elasticity, vol.128, pp.85-114.

[17] Tomar S. K. and Khurana A. (2018): Waves at the interface of dissimilar nonlocal micropolar elastic half-spaces.Mech. of ad. Mater. and struct., vol.26, pp.825-833.

[18] Tomar S.K. and Gogna M.L. (1992): Reflection and refraction of longitudinal wave micro-rotational at the interface between two micropolar elastic media in welded contact.- Int. J. Engng. Sci., vol.30, pp.1637-1646.

[19] Tomar S.K. and Gogna M.L. (1995): Reflection and refraction of longitudinal waves at the interface between two micropolar elastic media in welded contact.- J. Acoust. Soc. Am., vol.97, pp.822-830.

[20] Parfitt V.R. and. Eringen A.C. (1969): Reflection of plane waves from the flat boundary of a micropolar elastic halfspace.- J. Acoust. Soc. Am., vol.45, pp.1258-1272.

[21] Tomar S.K. (2015): Wave propagation in local and nonlocal microstretch elastic media.- Mec. of ad. Mater. and Struct., vol.84, pp.1-23.

[22] Singh D., Rani N. and Tomar S.K. (2016): Dilatational waves at a microstretch solid/fluid interface.- J. Vibr. Cont., vol.23, pp.3448-3467.

[23] Zhang P., Wei P. and Tang Q. (2015): Reflection of micropolar elastic waves at the non-free surface of a micropolar elastic half-space.- Acta Mech., vol.226, pp.2925-2937.

[24] Zhang P., Wei P. and Tang Q. (2015): Reflection of micropolar elastic waves at the non-free surface of a micropolar elastic half-space.- Acta mech., vol.226, pp.2925-2937.

[25] Zhang P., Wei P. and Tang Q. (2017): Reflection of longitudinal displacement wave at the visco-elastically supported boundary of a micropolar half space.- Meccanica, vol.52, pp.1641-1654.

[26] Achenbach J.D. (1973): Wave propagation in elastic solids.- North Holland, Amsterdam.

[27] Sarkar N. and Tomar S.K. (2019): Plane waves in nonlocal thermoelastic solid with voids.- J. of Thermal Stresses, vol.42, pp.580-606. 
[28] Sahrawat R.K., Poonam and Kumar K. (2020): Wave propagation in nonlocal couple stress thermoelastic solid.AIP Conf. Proceed., vol.2253, pp.1-14.

[29] Poonam, Sahrawat R.K., Kumar K. and Arti (2021): Plane wave propagation in functionally graded isotropic couple stress thermoelastic solid media under initial stress and gravity.- Eur. Phys. J. Plus, vol.136, pp.1-32.

[30] Sahrawat R.K., Poonam and Kumar K. (2021): Plane wave and fundamental solution in non-local couple stress micropolar thermoelastic solid without energy dissipation.- J. of Ther. Stress, vol.44, pp.295-314.

[31] Tomar S.K., Goyal N. and Szekers A. (2019): Plane waves in thermo-viscoelastic material with voids under different theories of thermoelasticity.- Int. J. of Applied Mechanics and Engineering, vol.24, No.3, pp.691-708.

[32] Kumar B., Kumar R. and Kaushal S. (2017): Viscosity effect on reflection and transmission coefficients between two micropolar viscothermoelastic half spaces with three-phase legs.- Int. J. of Eng. Sci. and Res. Tech., vol.9, pp.23482369.

Received: September 19, 2020

Revised: November 24, 2020 